\title{
Robust Parametric Tests of Constant Conditional Correlation in a MGARCH Model*
}

\author{
Wasel Shadat ${ }^{\dagger \ddagger}$ and Chris Orme \\ Economics, School of Social Sciences, University of Manchester, UK
}

September 17, 2015

\begin{abstract}
This paper provides a rigorous asymptotic treatment of new and existing asymptotically valid Conditional Moment testing procedures of the Constant Conditional Correlation assumption in a multivariate GARCH model. Full and partial Quasi Maximum Likelihood Estimation frameworks are considered, as is the robustness of these tests to non-normality. In particular, the asymptotic validity of the LM procedure proposed by Tse (2000) is analyzed and new asymptotically robust versions of this test are proposed for both estimation frameworks. A Monte Carlo study suggests that a robust Tse test procedure exhibits good size and power properties, unlike the original variant which exhibits size distortion under non-normality.
\end{abstract}

JEL classification: $\mathrm{C} 12$; $\mathrm{C} 32$

Keywords: Multivariate GARCH; Constant Conditional Correlation; Conditional Moment Tests; Robustness; Monte Carlo.

\section{Introduction}

Within a Multivariate GARCH (MGARCH) model, the conditional correlation approach has proved popular amongst applied workers when modelling volatility. Initially, the Constant Conditional Correlation (CCC) model was employed (see for example, Bollerslev (1990), Kroner and Claessens (1991), Kroner and Sultan (1991, 1993), Park and Switzer (1995) and Lien and Tse (1998)), whilst recently the Dynamic Conditional Correlation (DCC) model (Engle, 2002) has become more prevalent. Due to the simplicity and computational advantages of the CCC model, on the one hand, but the increased generality of the DCC approach, on the other, testing the adequacy of the CCC assumption within a MGARCH model remains important from both a practical and, therefore, theoretical point of view.

Indeed, the most widely used test of the CCC assumption, among applied workers, is Tse's (2000) LM test (see, for example, Lien, Tse and Tsui (2002), Andreou and Ghysels (2003), Lee (2006), Aslanidis, Osborn and Sensier (2008), among others) in preference to a number of other proposals in the literature

\footnotetext{
* An earlier version of the paper entitled 'An Investigation of Parametric Tests of the Constant Conditional Correlation Assumption' was presented at the European and Asian Meetings of the Econometric Society in Oslo (August 25-29, 2011) and Seoul (August 11-13, 2011). Detailed and exhaustive proofs are provided in a freely available on-line companion paper, Shadat \& Orme (2015). We are grateful for the insightful comments of the participants of these meetings and also to Ralf Becker, Alastair Hall and Len Gill. Two anonymous referees also provided constructive and insightful comments which were of enormous help in revising this paper. The standard disclaimer applies.

$\dagger$ Corresponding author: Dr Wasel Shadat, Economics, School of Social Science, University of Manchester, Manchester M13 9PL, UK. e-mail: waselbin.Shadat@manchester.ac.uk

¥The first author's research is part of his PhD thesis and was supported by a Commonwealth Scholarship and Fellowship Plan and a Manchester School Award; both of which are gratefully acknowledged.
} 
(e.g., Bollerslev (1990), Longin and Solnik (1995) and Bera and Kim (2002)) 円 Tse's (2000) procedure, unlike the information matrix test approach of Bera and Kim (2002), can be applied to high-dimensional data but it is predicated on a Full Quasi Maximum Likelihood Estimation (FQMLE) approach, together with an explicit assumption of normality when constructing the test statistic.

This paper addresses four inferential issues that emerge from Tse (2000): (i) Tse's Outer Product of the Gradient (OPG) version of the LM test is only guaranteed to be asymptotically valid under an explicit normality assumption. (ii) Even under normality the OPG variant of a LM test may demonstrate relatively poor finite sample performance; see, for example, Davidson and MacKinnon (1983), Bera and McKenzie (1986), Orme (1990), Chesher and Spady (1991). (iii) Tse's test procedure may not be robust to misspecification of the individual volatility (GARCH) equations. (iv) It is still common practice, when estimating a MGARCH model, to employ a two-stage or Partial Quasi Maximum Likelihood Estimation (PQMLE) approach, where the volatility parameters in each equation are first estimated using a univariate GARCH specification and, second, the correlation parameters are then estimated using these first-stage volatility parameter estimates (see Engle and Sheppard (2008), Hafner, Dijk and Franses (2005), Billio, Caporin and Gobbo (2006), among others); however, within this PQMLE framework, there appears to be no available test of the CCC assumption.

Thus, we propose, and provide a rigorous analysis of, asymptotically valid and non-normality robust tests of the CCC assumption, based on a Conditional Moment (CM) approach. These tests will be robust in the sense that their asymptotic validity does not depend on normality (unlike Tse (2002)); but they do require moment conditions which ensure standard asymptotic inferences can be applied. Such tests can be employed following either FQMLE or PQMLE and robust versions of Tse's test are given particular attention. The required derivations require some straightforward, yet tedious, algebraic results but lead to robust tests that are easy to implement. In our Monte Carlo study, with a moderate number of assets/time-series $(N=5)$, these tests demonstrate satisfactory size properties in most cases. Furthermore, whilst not addressed analytically, the Monte Carlo study also sheds some light on the robustness of the various test statistics to GARCH misspecifications in the individual volatility (GARCH) equations. From the panoply of procedures we consider, a robust version of Tse's LM test exhibits very good size and power properties under a variety of Data Generation Processes (DGPs).

The rest of this paper is organized as follows. The model, FQMLE, PQMLE and Tse's original LM test are reviewed in Section 2. In Section 3, a class of CM parametric tests is described, for both estimation frameworks, and robust variants proposed. This is extended in Section 4 to provide robust versions of Tse's LM test that can be employed following either FQMLE or PQMLE. Section 5 reports the findings of a Monte Carlo study and Section 6 concludes. The analysis follows standard first order asymptotic theory, but to avoid obfuscating the main issues, technical (but fairly standard) assumptions

\footnotetext{
${ }^{1}$ Nakatani and Teräsvirta (2009) proposed another LM test for volatility interaction where the null model is CCC GARCH model against the alternative of Extended CCC (ECCC) GARCH model.
} 
and proofs of the main results are relegated to an Appendix; with more detailed and exhaustive proofs provided in an accompanying freely available on-line paper, Shadat and Orme (2015), which also contains additional information concerning the Monte Carlo experiments undertaken.

The following notation is employed: the vec (.) operator stacks the $N$ columns of a $(M \times N)$ matrix as a $(M N \times 1)$ vector; vech (.) stacks the lower triangular portion of a $(N \times N)$ matrix as a $\left(\frac{1}{2} N(N+1) \times 1\right)$ vector; and, $\operatorname{vecl}($.$) stacks the strictly lower triangular portion of a (N \times N)$ matrix as a $\left(\frac{1}{2} N(N-1) \times 1\right)$ vector. Correspondingly, $s_{N}^{J}=\{(i, j): i=j, \ldots, N, j=1, \ldots, N$, and $i$ changing more quickly than $j\}$ defines the ordering of the elements of a $(N \times N)$ matrix $A=\left\{a_{i j}\right\}$ into $\operatorname{vech}(A)$ and $s_{N}^{C}=\{(i, j): i=j+1, \ldots, N, j=1, \ldots, N-1$, and $i$ changing more quickly than $j\}$ the corresponding ordering for the vecl (.) operator.

\section{The CCC Model and Tse's LM Test}

We consider the following standard CCC-GARCH linear regression specification

$$
y_{i t}=w_{i t}^{\prime} \varphi_{i}+\varepsilon_{i t}, \quad i=1, \ldots, N \quad t=1, \ldots, T,
$$

to model the $(N \times 1)$ time-series vector $y_{t}=\left\{y_{i t}\right\}$, with $T$ large and $N$ fixed/small, where $w_{i t}=$ $\left(y_{i, t-1}^{\prime}, d_{i t}^{\prime}\right)^{\prime}$ is the $(K \times 1)$ vector of regressor variables, containing current and lagged exogenous variables $\left(d_{i t}\right)$, and lagged dependent variables $\left(y_{i, t-1}\right)$ and $\varphi_{i} \subset \Re^{K}$ is an unknown vector of regression parameters. The volatility in the $(N \times 1)$ error vector $\varepsilon_{t}=\left\{\varepsilon_{i t}\right\}$ has a $\operatorname{GARCH}(p, q)$ specification of $h_{i t}=\alpha_{i 0}+\sum_{k=1}^{q} \alpha_{i k} \varepsilon_{i, t-k}^{2}+\sum_{j=1}^{p} \beta_{i j} h_{i, t-j}$ with $\eta_{i}=\left(\alpha_{i 0}, \alpha_{i 1}, \ldots, \alpha_{i q}, \beta_{i 1}, \ldots, \beta_{i p}\right)^{\prime} \subset \Re^{K^{*}}$ being an unknown vector of volatility parameters. The CCC model is described by

$$
\varepsilon_{t}=H_{t}^{1 / 2} \xi_{0 t}, \text { and } H_{t}=D_{t} \Gamma D_{t},
$$

where the $\xi_{0 t}$ are independently and identically distributed (iid) random vectors, with $E\left[\xi_{0 t}\right]=0$ and $E\left[\xi_{0 t} \xi_{0 t}^{\prime}\right]=I_{N}, D_{t}=\operatorname{diag}\left(h_{i t}^{1 / 2}\right)$ a $(N \times N)$ diagonal matrix and $\Gamma=\left\{\rho_{i j}\right\}$, a $(N \times N)$ time invariant symmetric positive definite matrix with $\rho_{i i}=1, i=1, \ldots, N$. Thus, the conditional covariance matrix, $H_{t}$, has elements $h_{i j t}=h_{i t}^{1 / 2} h_{j t}^{1 / 2} \rho_{i j}, i, j=1, \ldots, N$.

To be more precise about the parameterization employed, define $\theta_{i}=\left(\varphi_{i}^{\prime}, \eta_{i}^{\prime}\right)^{\prime} \subset \Re^{K+K^{*}}$ and $\omega=$ $\left(\theta^{\prime}, \rho^{\prime}\right)^{\prime} \in \Theta \subset \Re^{N^{*}}$, where $N^{*}=N\left(K+K^{*}\right)+\frac{1}{2} N(N-1)$ with $\theta=\left(\theta_{1}^{\prime}, \ldots, \theta_{N}^{\prime}\right)^{\prime} \subset \Re^{N\left(K+K^{*}\right)}$ and $\rho=\operatorname{vecl}(\Gamma) \bigsqcup^{2}$ Then, $\varepsilon_{i t}=y_{i t}-w_{i t}^{\prime} \varphi_{i} \equiv \varepsilon_{i t}\left(\varphi_{i}\right)$ with $h_{i t} \equiv h_{i t}\left(\theta_{i}\right), D_{t} \equiv D_{t}(\theta)$, and $H_{t} \equiv H_{t}(\omega)$. Letting $\omega_{0}=\left(\theta_{0}^{\prime}, \rho_{0}^{\prime}\right)^{\prime}$ denote the true parameter vector, with $\rho_{0}=\operatorname{vecl}\left(\Gamma_{0}\right)$, we have $\varepsilon_{0 t}=\varepsilon_{t}\left(\theta_{0}\right)$,

\footnotetext{
${ }^{2}$ For example, for a $\operatorname{AR}(1)$ CCC specification with $N=5$ and individual GARCH $(1,1)$ errors, we have $K=2, K^{*}=3$
} and $N^{*}=35$. 
$D_{0 t}=D_{t}\left(\theta_{0}\right)$, and $H_{0 t}=H_{t}\left(\omega_{0}\right)=D_{0 t} \Gamma_{0} D_{0 t}$ so that $E\left[\varepsilon_{0 t} \mid \mathcal{F}_{t-1}\right]=0$, and $E\left[\varepsilon_{0 t} \varepsilon_{0 t}^{\prime} \mid \mathcal{F}_{t-1}\right]=H_{0 t}$, where $\mathcal{F}_{t-1}=\sigma\left(\varepsilon_{0, t-1}, \varepsilon_{0, t-2}, \ldots\right) !^{3}$

Following Berkes, Horváth and Kokoszka (2003) and Ling and McAleer (2003), and given Assumption A3(i)(ii), the process for $h_{i t}$ has the representation $h_{i t}^{\infty}=\sum_{l=0}^{\infty} \psi_{i l} a_{i, t-l}$, where, for all $i, a_{i t}=\alpha_{i 0}+$ $\sum_{k=1}^{q} \alpha_{i k} \varepsilon_{i, t-k}^{2}$ and $\psi_{i l}=\sum_{j=1}^{p} \beta_{i j} \psi_{i, l-j}$ with $\psi_{i s}=0, s<0, \psi_{i 0}=1, \psi_{i l}>0, l>0$, and $0<$ $\sum_{l=0}^{\infty} \psi_{i l}=\left(1-\sum_{j=1}^{p} \beta_{i j}\right)^{-1}<\infty$. The coefficients, $\psi_{i l}$, decay exponentially fast, and there exist constants $\bar{K}>0$ and $0<\tau<1$, independent of $\omega$, such that $\psi_{i l} \leq \bar{K} \tau^{l}$, for all $i$. Then, Assumption $\mathrm{A}$ in the Appendix, ensures the identifiability, stationarity and ergodicity of the process $\left\{y_{i t}, \varepsilon_{0 i t}, h_{0 i t}\right\}$, where $h_{0 i t} \equiv h_{i t}^{\infty}\left(\theta_{i 0}\right)$; see Ling and McAleer (2003).

In the subsequent analyses, three alternative "transformed" error vectors are employed: volatility adjusted errors $\left(\zeta_{t}\right)$, "fully" standardized errors $\left(\xi_{t}\right)$ and (Tse's) transformed standardized errors $\left(\varepsilon_{t}^{*}\right)$. These are, respectively,

$$
\begin{aligned}
\zeta_{t} & \equiv \zeta_{t}(\theta)=D_{t}^{-1} \varepsilon_{t}=\left\{\zeta_{i t}\left(\theta_{i}\right)\right\} \\
\xi_{t} & \equiv \xi_{t}(\omega)=H_{t}^{-1 / 2} \varepsilon_{t}=\left\{\xi_{i t}(\omega)\right\} \\
\varepsilon_{t}^{*} & \equiv \varepsilon_{t}^{*}(\omega)=\Gamma^{-1} \zeta_{t}=\Gamma^{-1} D_{t}^{-1} \varepsilon_{t}=\left\{\varepsilon_{i t}^{*}(\omega)\right\}
\end{aligned}
$$

with $\zeta_{0 t}=\zeta_{t}\left(\theta_{0}\right), \xi_{0 t}=\xi_{t}\left(\omega_{0}\right), \varepsilon_{0 t}^{*}=\varepsilon_{t}^{*}\left(\omega_{0}\right)$, and satisfying: (i) $E\left[\zeta_{0 t} \mid \mathcal{F}_{t-1}\right]=0, E\left[\zeta_{0 t} \zeta_{0 t}^{\prime} \mid \mathcal{F}_{t-1}\right]=\Gamma_{0}$ (in the case of a CCC specification); (ii) $E\left[\xi_{0 t}\right]=0, E\left[\xi_{0 t} \xi_{0 t}^{\prime}\right]=I_{N}$, from $\left[2\right.$; ; and, (iii) $E\left[\varepsilon_{0 t}^{*} \mid \mathcal{F}_{t-1}\right]=0$, $E\left[\varepsilon_{0 t}^{*} \varepsilon_{0 t}^{* \prime} \mid \mathcal{F}_{t-1}\right]=\Gamma_{0}^{-1}$. For some estimator $\hat{\omega}=\left(\hat{\theta}^{\prime}, \hat{\rho}^{\prime}\right)^{\prime}$ of $\omega$, the estimated counterparts of 3 - (5) will be denoted $\hat{\zeta}_{t} \equiv \zeta_{t}(\hat{\theta}), \hat{\xi}_{t} \equiv \xi_{t}(\hat{\omega}), \hat{\varepsilon}_{t}^{*} \equiv \varepsilon_{t}^{*}(\hat{\omega})$ and similarly $\hat{\zeta}_{i t}, \hat{\xi}_{i t}$ and $\hat{\varepsilon}_{i t}^{*}$. Finally, where there is no ambiguity, this form of notation will be adopted for general functions of parameters $m_{t}(\omega)$; so that $m_{t} \equiv m_{t}(\omega), m_{0 t} \equiv m_{t}\left(\omega_{0}\right)$ and $\hat{m}_{t} \equiv m_{t}(\hat{\omega})$, etc.

\subsection{FQMLE and PQMLE Framework}

Given (1) and (2), the quasi-conditional log-likelihood per observation, $t$, is given by

$$
l_{t}^{*}(\omega)=-\frac{1}{2} \ln |\Gamma|-\frac{1}{2} \sum_{i=1}^{N} \ln h_{i t}-\frac{1}{2} \zeta_{t}^{\prime} \Gamma^{-1} \zeta_{t} .
$$

Assuming $\mathcal{L}_{T}^{*}(\omega)=T^{-1} \sum_{t=1}^{T} l_{t}^{*}(\omega)$ is twice continuously differentiable, and $\bar{g}_{T}^{*}(\omega)=T^{-1} \sum_{t=1}^{T} g_{t}^{*}(\omega)$, where $g_{t}^{*}(\omega)=\partial l_{t}^{*}(\omega) / \partial \omega$, then the FQML estimator, $\hat{\omega}=\arg \max _{\omega} \mathcal{L}_{T}^{*}(\omega)$, satisfies $\bar{g}_{T}^{*}(\hat{\omega})=0$. However, the observed $l_{t}^{*}(\omega)$ is constructed conditional on available pre-sample values, because $h_{i t}$ needs to be constructed recursively given initial values, $\varepsilon_{i 0}^{+}=\left(\varepsilon_{i 0}^{2}, \ldots, \varepsilon_{i, 1-q}^{2}, h_{i 0}, \ldots, h_{i, 1-p}\right)^{\prime}$. In order

\footnotetext{
${ }^{3}$ Given the context, there should be no confusion between the random vector $\varepsilon_{0 t}$, which has elements $\varepsilon_{0 i t}$, and the elements of $\varepsilon_{t}$, denoted $\varepsilon_{i t}, i=1, \ldots, N, t=1, \ldots, T$.
} 
to simplify the algebra and asymptotic theory, it is assumed (in addition) that the required pre-sample observations on $w_{i t}$ are also available and that $h_{i t}=0$ for all $i$ and $t \leq 0$. The simplifications derive from the fact that $h_{i t}$ can then be expressed as $h_{i t}=\sum_{l=0}^{t-1} \psi_{i l} a_{i, t-l}, t=1, \ldots, T$, but the processes $h_{i t}$ and $l_{t}^{*}(\omega)$ will not be stationary ergodic sequences $4^{4}$

Replacing $h_{i t}$ by $h_{i t}^{\infty}$ in $l_{t}^{*}(\omega)$, throughout, provides an unobserved but stationary and ergodic loglikelihood sequence

$$
l_{t}^{\infty *}(\omega)=-\frac{1}{2} \ln |\Gamma|-\frac{1}{2} \sum_{i=1}^{N} \ln h_{i t}^{\infty}-\frac{1}{2} \zeta_{t}^{\infty \prime} \Gamma^{-1} \zeta_{t}^{\infty},
$$

where $\zeta_{t}^{\infty}=\left\{\varepsilon_{i t} / \sqrt{h_{i t}^{\infty}}\right\}$. Then, with $N$ finite and letting $T \rightarrow \infty$, and under Assumptions A and B1, B2 described in the Appendix, $\hat{\omega} \stackrel{p}{\longrightarrow} \omega_{0}$ and $\sqrt{T}\left(\hat{\omega}-\omega_{0}\right) \stackrel{d}{\longrightarrow} N\left(0, J_{0}^{*-1} \Sigma_{g g}^{*} J_{0}^{*-1}\right)$, where $J_{0}^{*}=-E\left[\partial g_{t}^{\infty *}(\omega) / \partial \omega^{\prime}\right]_{\omega=\omega_{0}}$ and $\Sigma_{g g}^{*}=E\left[g_{t}^{\infty *}\left(\omega_{0}\right) g_{t}^{\infty *}\left(\omega_{0}\right)^{\prime}\right]$ are both finite and positive definite and $g_{t}^{\infty *}(\omega)=\partial l_{t}^{\infty *}(\omega) / \partial \omega$. That is to say, employing the recursively constructed $h_{i t}$ (rather than the "true" but unobserved $h_{i t}^{\infty}$ ) makes no difference asymptotically.

Adopting a PQMLE approach, and following Engle $(2002)$, we can write $\mathcal{L}_{T}^{*}(\omega)=\mathcal{L}_{T}(\theta)+\mathcal{L}_{T}^{C}(\omega)$ where $\mathcal{L}_{T}(\theta)=\frac{1}{T} \sum_{t=1}^{T} \sum_{i=1}^{N} l_{i t}\left(\theta_{i}\right)$, with $l_{i t}\left(\theta_{i}\right)=-\frac{1}{2}\left\{\ln h_{i t}+h_{i t}^{-1} \varepsilon_{i t}^{2}\right\}$ and $\mathcal{L}_{T}^{C}(\omega)=\frac{1}{T} \sum_{t=1}^{T} l_{t}^{C}(\omega)$, with $l_{t}^{C}(\omega)=-\frac{1}{2} \ln |\Gamma|-\frac{1}{2} \zeta_{t}^{\prime} \Gamma^{-1} \zeta_{t}+\frac{1}{2} \zeta_{t}^{\prime} \zeta_{t}$. Here, $\frac{1}{T} \sum_{t=1}^{T} l_{i t}\left(\theta_{i}\right)$ is the average log-likelihood for the $i^{t h}$ univariate GARCH regression model $(1)$ and $l_{t}^{C}(\omega)$ models the CCC structure. This affords a two-stage PQMLE procedure where at stage one we obtain $\tilde{\theta}_{i}=\arg \max _{\theta_{i}} \frac{1}{T} \sum_{t=1}^{T} l_{i t}\left(\theta_{i}\right)$, the consistent univariate GARCH QML estimators. Equivalently, $\tilde{\theta}=\arg \max _{\theta} \mathcal{L}_{T}(\theta)$, which satisfies $\bar{g}_{T}(\tilde{\theta})=0$, where $\bar{g}_{T}(\theta)=$ $\frac{1}{T} \sum_{t=1}^{T} g_{t}(\theta)$ and $g_{t}(\theta)=\sum_{i=1}^{N} \partial l_{i t}\left(\theta_{i}\right) / \partial \theta$, which is, of course, $g_{t}(\theta)=\left(\partial l_{1 t}\left(\theta_{1}\right) \partial \theta_{1}^{\prime}, \ldots, \partial l_{N t}\left(\theta_{N}\right) / \partial \theta_{N}^{\prime}\right)^{\prime}$. For the second stage, we employ all the first stage PQML estimator $\tilde{\theta}$ to obtain $\tilde{\rho}=\arg \max _{\rho} \mathcal{L}_{T}^{C}(\tilde{\theta}, \rho)$, which satisfies $\sum_{t=1}^{T}\left(\tilde{\varepsilon}_{i t}^{*} \tilde{\varepsilon}_{j t}^{*}-\tilde{\rho}^{i j}\right)=0, j<i$, where $\Gamma^{-1}=\left\{\rho^{i j}\right\}$. The resulting PQML estimator, $\tilde{\omega}=\left(\tilde{\theta}^{\prime}, \tilde{\rho}^{\prime}\right)^{\prime}$, is consistent, but asymptotically inefficient relative to FQMLE 5 If we adopt Bollerslev's (1990) alternative parameterization of $l_{i t}\left(\theta_{i}\right)$, it transpires that the ensuing $\tilde{\rho}$ has a closed form expression satisfying $\sum_{t=1}^{T}\left(\tilde{\zeta}_{i t} \tilde{\zeta}_{j t}-\tilde{\rho}_{i j}\right)=0, j<i$. Even without this alternative parameterization, it is still the case that the simple estimator $\tilde{\rho}_{i j}=\frac{1}{T} \sum_{t=1}^{T} \tilde{\zeta}_{i t} \tilde{\zeta}_{j t}, j<i$ will be consistent for the true correlation parameter value, and this will be the estimator, together with $\tilde{\theta}_{i}$, that we shall employ in the PQMLE framework. Moreover, it turns out that the limit distributions of the various test indicators that we shall consider, obtained from PQMLE, are not influenced by this choice of $\tilde{\rho}$ and this leads to the construction of relatively simple asymptotically valid test statistics. Thus, we just need the separate limit distributions of $\sqrt{T}\left(\tilde{\theta}_{i}-\theta_{i 0}\right)$ and Assumptions A and B1, B2, in the Appendix, imply $\sqrt{T}\left(\tilde{\theta}-\theta_{0}\right) \stackrel{d}{\rightarrow} N\left(0, J_{0}^{-1} \Sigma_{g g} J_{0}^{-1}\right)$ where the (block diagonal) matrix

\footnotetext{
${ }^{4}$ Note, that this is not the same start-up scheme employed by either Ling and McAleer $(2003)$, who choose $\varepsilon_{i 0}^{+}=\mathbf{0}$, Berkes et al (2003), or Francq and Zakoian (2004). In practice, and for all inferential procedures described in this paper, any constant value can be chosen for $\varepsilon_{i 0}^{+}$, in order to generate $h_{i t}, t=1, \ldots, T$.

${ }^{5}$ Hafner and Herwartz (2008) provided an analytical expression for the asymptotic variance of the PQML estimator, for both the CCC and DCC models.
} 
$J_{0}=-E\left[\partial g_{t}^{\infty}\left(\theta_{0}\right) / \partial \theta^{\prime}\right]=\operatorname{diag}\left(-E\left[\partial^{2} l_{i t}^{\infty}\left(\theta_{i 0}\right) / \partial \theta_{i} \partial \theta_{i}^{\prime}\right]\right)$ and $\Sigma_{g g}=E\left[g_{t}^{\infty}\left(\theta_{0}\right) g_{t}^{\infty}\left(\theta_{0}\right)^{\prime}\right]$ are both finite and positive definite, and $g_{t}^{\infty}(\theta)=\sum_{i=1}^{N} \partial l_{i t}^{\infty}\left(\theta_{i}\right) / \partial \theta$ with $l_{i t}^{\infty}\left(\theta_{i}\right)=-\frac{1}{2}\left\{\ln h_{i t}^{\infty}+\varepsilon_{i t}^{2} / h_{i t}^{\infty}\right\}$; see, e.g., Halunga and Orme (2009, Theorem 1).

\subsection{Tse's LM Test of the CCC Assumption}

For the purposes of constructing Tse's test, a dynamic correlation structure of the form $\rho_{i j t}=\rho_{i j}+$ $\gamma_{i j} \zeta_{i, t-1} \zeta_{j, t-1}$ is assumed, where $\gamma_{i i}=0, \gamma_{i j}=\gamma_{j i}$, even though $\rho_{i j t}$ is not a well-defined alternative to the CCC since $\Gamma_{t}=\left\{\rho_{i j t}\right\}$ is not necessarily a positive definite matrix for all $t$. There are $\frac{N(N-1)}{2}$ additional parameters in DCC "alternative" model with the null hypothesis of CCC being $H_{0}: \gamma_{i j}=0$, for all distinct $i, j$, and $i>j$. Tse (2000) employed the Lagrange Multiplier (LM) principle and proposed an OPG variant of the LM test statistic which, in this case with test variables $\hat{\zeta}_{t-1} \hat{\zeta}_{t-1}^{\prime}$, is

$$
\widehat{L M}_{T}^{*}=\iota_{T}^{\prime} \hat{\Xi}^{*}\left(\hat{\Xi}^{* \prime} \hat{\Xi}^{*}\right)^{-1} \hat{\Xi}^{* \prime} \iota_{T}
$$

where $\hat{\Xi}^{*}$ is a $\left(T \times N^{*}+\frac{N(N-1)}{2}\right)$ matrix, with rows equal to $\left(\hat{g}_{t}^{* \prime}, \operatorname{vecl}\left(\left(\hat{\varepsilon}_{t}^{*} \hat{\varepsilon}_{t}^{* \prime}-\hat{\Gamma}^{-1}\right) \odot \hat{\zeta}_{t-1} \hat{\zeta}_{t-1}^{\prime}\right)^{\prime}\right), \odot$ denotes the Hadamard product, and $\iota_{T}$ is the $(T \times 1)$ column vector of ones: 7 Under the usual regularity conditions $\widehat{L M}_{T}^{*}$ is asymptotically distributed as $\chi_{\frac{N(N-1)}{2}}^{2}$.

The notation $\widehat{L M}_{T}^{*}$ is used to emphasize that $(6)$ is constructed from $\hat{\omega}$ and cannot be implemented directly using $\tilde{\omega}$. Furthermore, the OPG construction advocated by Tse (2000) may be sensitive to non-normality, and some evidence for this is provided by Tse (2000, Section 5). In the next section we develop a Conditional Moment (CM) testing framework of the CCC assumption which accommodates Tse's Test. This framework provides, in Section 4 , non-normality robust Tse test procedures for both the FQMLE and PQMLE cases. However, for this particular choice of test variables, $\zeta_{i, t-1} \zeta_{j, t-1}$, a stronger moment condition of $E\left|\varepsilon_{0 i t}\right|^{8}<\infty$ for all $i, t$, is then required in order to justify the asymptotic validity of these robust tests.

\section{A Class of Asymptotically Valid CM Test Procedures}

If the CCC specification is correct, then $E\left[\zeta_{0 t} \zeta_{0 t}^{\prime}-\Gamma_{0} \mid \mathcal{F}_{t-1}\right]=0$ in which $h_{0 i t}=h_{i t}^{\infty}\left(\theta_{i 0}\right)$. The diagonal elements of $\left(\zeta_{t} \zeta_{t}^{\prime}-\Gamma\right)$ correspond to the individual GARCH (or volatility) specifications, whereas the off-diagonal elements correspond to the CCC assumption. Also due to the symmetry there are $\frac{1}{2} N(N+1)$ independent (distinct) restrictions in this moment condition; i.e., $E\left[v_{0 t}^{J} \mid \mathcal{F}_{t-1}\right]=0$, where $v_{0 t}^{J}=\operatorname{vech}\left(\zeta_{0 t} \zeta_{0 t}^{\prime}-\Gamma_{0}\right)$, the superscript $J$ indicating joint testing of both the CCC and of the individual

\footnotetext{
${ }^{6}$ Here, cross-products of lagged standardised "residuals", are employed as "test variables" which is feasible using the LM principle and, since this "alternative" is an artificial device simply employed to construct a test statistic, Silvennoinen and Teräsvirta (2009) interpreted this as a general misspecification test.

${ }^{7}$ Details of $g_{t}^{\infty *}(\omega)$, and hence $g_{t}^{*}(\omega)$, are given in the Appendix, Proposition 6
} 
volatility specifications. The typical element of this moment condition can be written as

$$
E\left[\zeta_{0, i t} \zeta_{0, j t}-\rho_{0, i j} \mid \mathcal{F}_{t-1}\right]=0, i \geq j, \quad i=2, \ldots, N
$$

When the underlying moment restriction is (7), the ensuing test will be referred as the Full CM (FCM) test and can be treated as a joint misspecification test of the complete MGARCH error specification. If we are only interested in testing the CCC assumption, the moment condition is $E\left[v_{0 t}^{C} \mid \mathcal{F}_{t-1}\right]=0$, where $v_{0 t}^{C}=\operatorname{vecl}\left(\zeta_{0 t} \zeta_{0 t}^{\prime}-\Gamma_{0}\right)$ and the superscript $C$ denotes testing only the CCC assumption; i.e.,

$$
E\left[\zeta_{0, i t} \zeta_{0, j t}-\rho_{0, i j} \mid \mathcal{F}_{t-1}\right]=0, i>j, \quad i=2, \ldots, N
$$

The ensuing test based on (8) will be referred to as the CCC CM (CCM) test.

The implication of (7) is that misspecification tests of the CCC model can be constructed as tests of the following moment conditions

$$
E\left[\left(\zeta_{0, i t} \zeta_{0, j t}-\rho_{0, i j}\right) r_{i j, t}\left(\omega_{0}\right)\right]=0,
$$

where the $\left(q_{i j} \times 1\right)$ vector $r_{i j, t}\left(\omega_{0}\right)$ is a $\mathcal{F}_{t-1}$ measurable function, possibly depending upon the processes $h_{0 i t}$ and $h_{0 j t}$ 8 $^{8} \mathrm{~A} \mathrm{CM}$ test indicator vector can then be constructed, up to a knowledge of $\omega$, as $\bar{m}_{T}(\omega)=$ $\frac{1}{T} \sum_{t=1}^{T} m_{t}(\omega)$ with the vector $m_{t}(\omega)$ constructed from the "stacked" sub-vectors

$$
m_{i j, t}(\omega)=\left(\zeta_{i t} \zeta_{j t}-\rho_{i j}\right) r_{i j, t}=v_{i j, t} r_{i j, t}, \quad\left(q_{i j} \times 1\right)
$$

where $v_{i j, t}=\left(\zeta_{i t} \zeta_{j t}-\rho_{i j}\right)$, a scalar, and $r_{i j, t} \equiv r_{i j, t}(\omega)$. Note that the $m_{i j, t}(\omega)$ are arranged in $m_{t}(\omega)$, ordered by $(i, j)$, according to either $s_{N}^{J}$ (as in the vech(.) operator) or $s_{N}^{C}$ (as in the vecl(.) operator). In the former case, this will be denoted $\bar{m}_{T}^{J}(\omega)=\frac{1}{T} \sum_{t=1}^{T} m_{t}^{J}(\omega), q^{J} \times 1$, where $q^{J}=\sum_{i>j} q_{i j}$, whilst in the latter case it will be $\bar{m}_{T}^{C}(\omega)=\frac{1}{T} \sum_{t=1}^{T} m_{t}^{C}(\omega), q^{C} \times 1$, where $q^{C}=\sum_{i>j} q_{i j}=q^{J}-\sum_{i} q_{i i}$. Then, $\bar{m}_{T}^{J}(\omega)$ will be referred to as the FCM test indicator, $\bar{m}_{T}^{C}(\omega)$ the CCM test indicator and both can be constructed employing either the FQMLE, $\hat{\omega}$, or the PQMLE, $\tilde{\omega}$.

The following specials cases emerge:

1. If $r_{t}$ is a common vector of test variables employed for all $i, j$, then $\bar{m}_{T}^{J}(\omega)=\frac{1}{T} \sum_{t=1}^{T} v_{t}^{J} \otimes r_{t}$ and $\bar{m}_{T}^{C}(\omega)=\frac{1}{T} \sum_{t=1}^{T} v_{t}^{C} \otimes r_{t}$, where $v_{t}^{J}=\operatorname{vech}\left(\zeta_{t} \zeta_{t}^{\prime}-\Gamma\right)$ and $v_{t}^{C}=\operatorname{vecl}\left(\zeta_{t} \zeta_{t}^{\prime}-\Gamma\right)$.

2. If $r_{i j, t}$ is a scalar, with $\Upsilon_{t}=\left\{r_{i j, t}\right\},(N \times N)$, then $\bar{m}_{T}^{J}(\omega)=\frac{1}{T} \sum_{t=1}^{T} v_{t}^{J} \odot r_{t}^{J}$ and $\bar{m}_{T}^{C}(\omega)=$ $\frac{1}{T} \sum_{t=1}^{T} v_{t}^{C} \odot r_{t}^{C}$, where $r_{t}^{J}=\operatorname{vech}\left(\Upsilon_{t}\right), r_{t}^{C}=\operatorname{vecl}\left(\Upsilon_{t}\right)$.

${ }^{8}$ Although $\mathcal{F}_{t-1}$ measurable, we write $r_{i j, t}$ in defining $r_{i j, t}\left(\omega_{0}\right)$ rather than, say, $r_{i j, t-1}$. This is consistent with the usual notation $h_{0 i t}$, which is also $\mathcal{F}_{t-1}$ measurable. 
Tse's LM test can be interpreted as a test of the moment condition $E\left[\operatorname{vecl}\left(\varepsilon_{0 t}^{*} \varepsilon_{0 t}^{* \prime}-\Gamma_{0}^{-1} \mid \mathcal{F}_{t-1}\right)\right]=0$, where $\varepsilon_{t}^{*}$ is given in 5 . Since $\varepsilon_{t}^{*} \varepsilon_{t}^{* \prime}-\Gamma^{-1}=\Gamma^{-1}\left(\zeta_{t} \zeta_{t}^{\prime}-\Gamma\right) \Gamma^{-1}$, the $(k, l)^{t h}$ element of this $(N \times N)$ matrix is $\rho^{k \prime}\left(\zeta_{t} \zeta_{t}^{\prime}-\Gamma\right) \rho^{l}=\operatorname{vec}\left(\rho^{k} \rho^{l \prime}\right)^{\prime} D_{N} \operatorname{vech}\left(\zeta_{t} \zeta_{t}^{\prime}-\Gamma\right)$, where $\rho^{k}$ is the $k^{t h}$ column of $\Gamma^{-1}$ and $D_{N}$ is the $\left(N^{2} \times \frac{1}{2} N(N+1)\right)$ duplication matrix ${ }^{9}$ Exploiting the properties of $D_{N}$, we can write $\varepsilon_{k t}^{*} \varepsilon_{l t}^{*}-\rho^{k l}=\pi_{k l}^{\prime} v_{t}^{J}$, where $\pi_{k l}=\operatorname{vech}\left(\rho^{k} \rho^{l \prime}+\rho^{k} \rho^{l \prime}-\operatorname{dg}\left(\rho^{k} \rho^{l \prime}\right)\right),\left(\frac{1}{2} N(N+1) \times 1\right)$. For example, for $N=2$ we have $\rho_{12}=\rho_{21} \equiv \rho$, say, and $\pi_{21}^{\prime} \equiv \pi^{\prime}=\frac{1}{\left(1-\rho^{2}\right)^{2}}\left[\begin{array}{ccc}-\rho & 1+\rho^{2} & -\rho\end{array}\right]$.

Since $\pi_{k l}^{\prime} v_{t}^{J}$ is a scalar, the $(k, l)^{t h}$ element of a Tse (LM test) indicator employing arbitrary test variables, $\phi_{k l, t},\left(q_{k l} \times 1\right)$, with indices $(k, l)$ ordered according to $s_{N}^{C}$, can be expressed as

$$
\bar{m}_{k l, T}^{L M}(\omega)=\frac{1}{T} \sum_{t=1}^{T}\left\{\pi_{k l}^{\prime} v_{t}^{J}\right\} \phi_{k l, t}=\left(\pi_{k l}^{\prime} \otimes I_{q_{k l}}\right) \bar{m}_{(k l) T}^{J}(\omega), \quad\left(q_{k l} \times 1\right)
$$

where $\bar{m}_{(k l) T}^{J}(\omega)=\frac{1}{T} \sum_{t=1}^{T} v_{t}^{J} \otimes \phi_{k l, t},\left(\frac{q_{k l}}{2} N(N+1) \times 1\right)$, is a joint FCM test indicator vector, of the form $\bar{m}_{T}^{J}(\omega)$, but constructed with a common $\left(q_{k l} \times 1\right)$ vector of test variables, $\phi_{k l, t}$, for all elements of $v_{t}^{J}$. Therefore Tse's test indicator is accommodated in this CM framework since its $(k, l)^{t h}$ element is simply the linear combination (through $\pi_{k l}^{\prime} \otimes I_{q_{k l}}$ ) of $A L L$ the FCM test indicators. Recall that in Tse's original test, Section $2.2, \phi_{k l, t}=\zeta_{k, t-1} \zeta_{l, t-1}$, and $q_{k l}=1$.

To construct asymptotically valid CM tests of the CCC hypothesis we need to establish the limit distributions of the test indicator vectors. This is done in the following two sections for both the FQMLE and PQMLE cases, for which we need to introduce some more notation. Let $\sqrt{T} \bar{m}_{T}(\omega)=$ $\frac{1}{\sqrt{T}} \sum_{t=1}^{T} m_{t}(\omega)$ denote either $\sqrt{T} \bar{m}_{T}^{J}(\omega)$ or $\sqrt{T} \bar{m}_{T}^{C}(\omega)$, according to the test under consideration, where $m_{t} \equiv m_{t}(\omega),(q \times 1)$ denotes either $m_{t}^{J}(\omega)$ or $m_{t}^{C}(\omega)$ as defined above (with $q=q^{J}$, or $q=q^{C}$, respectively) and the vector $v_{0 t} \equiv v_{t}\left(\omega_{0}\right)$ will denote either $v_{0 t}^{J}$, or $v_{0 t}^{C}$. In particular, $m_{0 t} \equiv m_{t}\left(\omega_{0}\right)$ is constructed from the stacked $\left(q_{i j} \times 1\right)$ sub-vectors, $m_{i j, t}\left(\omega_{0}\right)$. We shall also employ the following at various times: $z_{i t}^{\prime}=\frac{1}{h_{i t}} \frac{\partial h_{i t}}{\partial \theta_{i}^{\prime}}=\left(c_{i t}^{\prime}, x_{i t}^{\prime}\right)$, where $c_{i t}^{\prime}=\frac{1}{h_{i t}} \frac{\partial h_{i t}}{\partial \varphi_{i}^{\prime}}$ and $x_{i t}^{\prime}=\frac{1}{h_{i t}} \frac{\partial h_{i t}}{\partial \eta_{i}^{\prime}}$, and $f_{i t}^{\prime}=\left(w_{i t}^{\prime} / \sqrt{h_{i t}}, 0_{K^{*}}^{\prime}\right)$, where $0_{K^{*}}$ is the $\left(K^{*} \times 1\right)$ vector of zeroes. Finally, and similarly to $l_{t}^{\infty *}(\omega)$ and $g_{t}^{\infty *}(\omega)$, a superscript of $\infty$ signifies that $h_{i t}\left(\right.$ and/or $\left.h_{j t}\right)$ has been replaced by $h_{i t}^{\infty}\left(\right.$ and/or $\left.h_{j t}^{\infty}\right)$ where necessary.

\subsection{Tests based on FQMLE}

An asymptotically valid $\chi^{2}$ test statistic, and test procedure, is justified by the following results:

Proposition 1 Suppose Assumptions $A$ and $B$, as described in the Appendix, hold. Then, $\Sigma^{*}=$ $E\left[u_{t}^{\infty *}\left(\omega_{0}\right) u_{t}^{\infty *}\left(\omega_{0}\right)^{\prime}\right]$ is finite, where $u_{t}^{\infty *}(\omega)^{\prime}=\left(m_{t}^{\infty}(\omega)^{\prime}, g_{t}^{\infty *}(\omega)^{\prime}\right)$. Furthermore:

(i) $\frac{1}{\sqrt{T}} \sum_{t=1}^{T} u_{t}^{\infty *}\left(\omega_{0}\right) \stackrel{d}{\rightarrow} N\left(0, \Sigma^{*}\right)$, where $\Sigma^{*}=E\left[u_{t}^{\infty *}\left(\omega_{0}\right) u_{t}^{\infty *}\left(\omega_{0}\right)^{\prime}\right]$ is finite; and,

\footnotetext{
${ }^{9}$ For $A=A^{\prime}, D_{N} \operatorname{vech}(A)=\operatorname{vec}(A)$ whilst for any $A, D_{N}^{\prime} \operatorname{vec}(A)=\operatorname{vech}\left(A+A^{\prime}-\operatorname{dg}(A)\right)$, where $\operatorname{dg}(A)$ forms the diagonal matrix from the diagonal elements of the square matrix $A$; see Magnus and Neudecker (1986).
} 
(ii) $\frac{1}{T} \sum_{t=1}^{T} u_{t}^{*}(\hat{\omega}) u_{t}^{*}(\hat{\omega})^{\prime}-\Sigma^{*}=o_{p}(1)$, for any $\hat{\omega}-\omega_{0}=o_{p}(1)$, where $u_{t}^{*}(\omega)^{\prime}=\left(m_{t}(\omega)^{\prime}, g_{t}^{*}(\omega)^{\prime}\right)$.

Remark 1 The choice of test variables $r_{i j, t}=\frac{\varepsilon_{i, t-1} \varepsilon_{j, t-1}}{\sqrt{h_{i, t-1}} \sqrt{h_{j, t-1}}}$ requires a strengthening of B1, in the Appendix, to $E\left|\varepsilon_{0 i t}\right|^{8}<\infty$. In the case of $\bar{m}_{T}^{J}\left(\omega_{0}\right)$, for example, $\Sigma_{m m} \equiv E\left[m_{t}^{\infty}\left(\omega_{0}\right) m_{t}^{\infty}\left(\omega_{0}\right)^{\prime}\right]$ is block partitioned with $\left(q_{i j} \times q_{k l}\right)$ blocks $E\left[v_{i j, t}^{\infty} v_{k l, t}^{\infty} r_{i j, t}^{\infty} r_{k l, t}^{\infty \prime}\right]_{\omega=\omega_{0}}$, with both $(i, j)$ and $(k, l)$ ordered according to $s_{N}^{J}$. The modification is obvious for $\bar{m}_{T}^{C}\left(\omega_{0}\right)$ which simply removes all entries $v_{i i, t}^{\infty 2} r_{i i, t}^{\infty} r_{i i, t}^{\infty \prime}, i=1, \ldots, N$, from $\Sigma_{m m}$. The following specials cases emerge:

1. If $r_{t}$ is the same vector of test variables employed for all $i, j$, then $\Sigma_{m m}=E\left[v_{0 t}^{\infty} v_{0 t}^{\infty \prime} \otimes r_{0 t}^{\infty} r_{0 t}^{\infty \prime}\right]$.

2. If $r_{i j, t}$ is a scalar, with $r_{t}$ being either $r_{t}^{J}$ or $r_{t}^{C}$, as appropriate. Then, $\Sigma_{m m}=E\left[r_{0 t}^{\infty} r_{0 t}^{\infty \prime} \odot v_{0 t}^{\infty} v_{0 t}^{\infty \prime}\right]$.

Proposition 2 Under the assumptions of Proposition $1, \sqrt{T} \bar{m}_{T}(\hat{\omega}) \stackrel{d}{\longrightarrow} N\left(0, V^{*}\right)$, where $V^{*}=A_{0}^{*} \Sigma^{*} A_{0}^{* \prime}$, and $A_{0}^{*}=\left[I_{q}:-B_{0}^{*} J_{0}^{*-1}\right]$, with $J_{0}^{*}=-E\left[\frac{\partial g_{t}^{\infty *}\left(\omega_{0}\right)}{\partial \omega^{\prime}}\right]$, positive definite, $B_{0}^{*}=-E\left[\frac{\partial m_{t}^{\infty}\left(\omega_{0}\right)}{\partial \omega^{\prime}}\right]$, and $I_{q}$ is the $(q \times q)$ identity matrix.

In the case of $\bar{m}_{T}^{J}(\hat{\omega})$, for example, $B_{0}^{*}$ is block partitioned as $B_{0}^{*}=\left[B_{0, i j}^{*}\right]$, with blocks $B_{0, i j}^{*}$ stacked (vertically) ordered by $(i, j)$, according to $s_{N}^{J}$, and given by

$$
B_{0, i j}^{*}=-E\left[r_{i j, t}^{\infty} \frac{\partial\left(\zeta_{i t}^{\infty} \zeta_{j t}^{\infty}-\rho_{i j}\right)}{\partial \omega^{\prime}}\right]_{\omega=\omega_{0}}
$$

noting that $\partial m_{i j, t}^{\infty} / \partial \omega^{\prime}=r_{i j, t}^{\infty}(\omega) \partial v_{i j, t}^{\infty} / \partial \omega^{\prime}+v_{i j, t}^{\infty} \partial r_{i j, t}^{\infty}(\omega) / \partial \omega^{\prime}$, and $E\left[v_{i j, t}^{\infty}\left(\omega_{0}\right) \mid \mathcal{F}_{t-1}\right]=0$, so that $E\left[v_{i j, t}^{\infty} \partial r_{i j, t}^{\infty}(\omega) / \partial \omega^{\prime} \mid \mathcal{F}_{t-1}\right]_{\omega=\omega_{o}}=0$. The modification is obvious for $\bar{m}_{T}^{C}(\hat{\omega})$ and simply removes all $B_{i i}^{*}$ blocks, and in the special case that $r_{t}$ is the same vector of test variables employed for all $i, j$, then $B_{0}^{*}=-E\left[\partial v_{t}^{\infty}\left(\omega_{0}\right) / \partial \omega^{\prime} \otimes r_{0 t}^{\infty}\right]$.

From Proposition 2, and provided $V^{*}$ is positive definite, the general form of the test statistic is

$$
\hat{S}_{T}^{*}=T \bar{m}_{T}(\hat{\omega})^{\prime}\left\{\hat{V}_{T}^{*}\right\}^{-1} \bar{m}_{T}(\hat{\omega})
$$

which has a limit $\chi_{q}^{2}$ distribution, under the null, where $\hat{V}_{T}^{*}$ is any consistent estimator for $V^{*}$.

To construct asymptotically valid test statistics we need consistent estimators for $V^{*}$. In doing so, we consider the cases of Gaussian and non-Gaussian distributions, respectively, for the fully standardized error process, $\xi_{0 t}$. The first case provides the well-known OPG covariance matrix estimator, denoted $\hat{V}_{T}^{*(o)}$. For the more general case, we develop a non-normality robust procedure, in the similar spirit of Wooldridge $\left(1 9 9 0 \longdiv { 1 0 }\right.$ built on a robust variance-covariance matrix estimator denoted $\hat{V}_{T}^{*(r)}$. This estimator will be robust in the sense that its consistency asymptotic does not depend on normality, but it does require moment conditions which ensure standard asymptotic inferences can be applied.

\footnotetext{
${ }^{10}$ Similar approach was employed by Halunga and Orme (2009).
} 


\subsubsection{The OPG-FQMLE Test}

Define the $(T \times q)$ matrix $M \equiv M(\omega)$ to have rows $m_{t}(\omega)^{\prime}$ and $G^{*} \equiv G^{*}(\omega)$ is a $\left(T \times N^{*}\right)$ matrix with rows $g_{t}^{*}(\omega)^{\prime}=\frac{\partial l_{t}^{*}(\omega)}{\partial \omega^{\prime}}$, with the understanding that $\hat{M} \equiv M(\hat{\omega})$ and $\hat{G} \equiv G(\hat{\omega})$. By Proposition 6(ii) and (iv), Lemma 1 implies that a consistent estimator for $\Sigma^{*}$ is $T^{-1} \hat{U}^{* \prime} \hat{U}^{*}=\frac{1}{T} \sum_{t=1}^{T} u_{t}^{*}(\hat{\omega}) u_{t}(\hat{\omega})^{\prime}$, where $\hat{U}^{*}=\left(\hat{M}, \hat{G}^{*}\right)$ has rows $u_{t}^{* \prime}(\omega)=\left(m_{t}^{\prime}(\omega), g_{t}^{* \prime}(\omega)\right)^{\prime}$. However, under the additional assumption of normality, $\xi_{0 t} \sim N\left(0, I_{N}\right)$, the generalized IM inequality holds (Newey, 1985) so that consistent estimators of $J_{0}^{*}$ and $B_{0}^{*}$ will be $T^{-1} \hat{G}^{* \prime} \hat{G}^{*}$ and $T^{-1} \hat{G}^{* \prime} \hat{M}$, respectively. In this case, a consistent estimator for $V^{*}$ can be obtained as

$$
\hat{V}_{T}^{*(o)}=T^{-1}\left(\hat{M}^{\prime} \hat{M}-\hat{M}^{\prime} \hat{G}^{*}\left(\hat{G}^{* \prime} \hat{G}^{*}\right)^{-1} \hat{G}^{* \prime} \hat{M}\right) .
$$

This provides the well-known OPG form formulation of $(12)$ as

$$
\hat{S}_{T}^{*(o)}=\iota_{T}^{\prime} \hat{U}^{*}\left(\hat{U}^{* \prime} \hat{U}^{*}\right)^{-1} \hat{U}^{* \prime} \iota_{T}
$$

which is simply of the form $T \times R_{u}^{2}$, where $R_{u}^{2}$ is the (uncentred) $R^{2}$ coefficient following a regression of $\iota_{T}$ on $\hat{U}^{*}$.

\subsubsection{The Robust-FQMLE Test}

Here we construct a (non-normality) robust estimator for $V^{*}=A_{0}^{*} \Sigma^{*} A_{0}^{* \prime}$, noting from above that $T^{-1} \hat{U}^{* \prime} \hat{U}^{*}-\Sigma^{*}=o_{p}(1)$, but without necessarily assuming normality. A robust estimator for $A_{0}^{*}$ requires robust estimators of $J_{0}^{*}=-E\left[\frac{\partial^{2} l_{t}^{\infty *}\left(\omega_{0}\right)}{\partial \omega \partial \omega^{\prime}}\right]$ and $B_{0}^{*}=-E\left[\frac{\partial m_{t}^{\infty}\left(\omega_{0}\right)}{\partial \omega^{\prime}}\right]$. The strategy for construction of such estimators follows, e.g., Nakatani and Teräsvirta (2009): define the matrix $J_{T}^{*}(\omega)$, which is constructed as $-\frac{1}{T} \sum_{t=1}^{T} E\left[\frac{\partial^{2} l_{t}^{\infty *}\left(\omega_{0}\right)}{\partial \omega \partial \omega^{\prime}} \mid \mathcal{F}_{t-1}\right]$ but, once conditional expectations have been taken, $\omega$ replaces $\omega_{0}$ and $h_{i t}$ replaces $h_{i t}^{\infty}$. Similarly, $B_{T}^{*}(\omega)$ is constructed from $-\frac{1}{T} \sum_{t=1}^{T} E\left[\frac{\partial m_{t}^{\infty}\left(\omega_{0}\right)}{\partial \omega^{\prime}} \mid \mathcal{F}_{t-1}\right]$ in the same way. We introduce the following additional notation: (i) $Z_{i}$ is a $\left(T \times K+K^{*}\right)$ matrix having rows $z_{i t}^{\prime}=\frac{1}{h_{i t}} \frac{\partial h_{i t}}{\partial \theta_{i}^{\prime}}=\left(c_{i t}^{\prime}, x_{i t}^{\prime}\right)$; (ii) $F_{i}$ is the $\left(T \times K+K^{*}\right)$ matrix with rows $f_{i t}^{\prime}=\left(w_{i t}^{\prime} / \sqrt{h_{i t}}, 0_{K^{*}}^{\prime}\right)$; (iii) $R_{i j}$ is the $\left(T \times q_{i j}\right)$ matrix having rows $r_{i j, t}^{\prime}, t=1, \ldots, T$; (iv) $Z=\operatorname{diag}\left(Z_{i}\right)$ and $F=\operatorname{diag}\left(F_{i}\right)$ are $\left(T N \times N\left(K+K^{*}\right)\right)$ block-diagonal matrices; $(\mathrm{v}) e_{i}$ is the $i^{t h}$ column of the $I_{N}$, the $(N \times N)$ identity matrix, and $e_{i j}=\operatorname{vecl}\left(\left(1-\delta_{i j}\right) e_{i} e_{j}^{\prime}\right)$, so that $e_{i i}$ is a vector of zeros, for all $i=1, \ldots, N ; E_{N}$ is the $\left(N^{2} \times N\right)$ matrix, with columns $e_{i} \otimes e_{i}$ and $L_{N}$ is the $\left(N^{2} \times \frac{1}{2} N(N-1)\right)$ matrix with columns $\left(e_{i} \otimes e_{j}\right)+\left(e_{j} \otimes e_{i}\right)$, ordered by $(i, j)$ according to $s_{N}^{C} ;\left(\right.$ vi) $\Gamma_{A}=I_{N}+\left(\Gamma^{-1} \odot \Gamma\right)=\Gamma_{A}^{\prime},(N \times N)$; and, (vii) $P=I_{N} \otimes \Gamma^{-1}+\Gamma^{-1} \otimes I_{N}=P^{\prime},\left(N^{2} \times N^{2}\right)$.

Then we have the following result:

Proposition 3 Under the Assumptions of Proposition 6, in the Appendix 
(i) $J_{T}^{*}(\hat{\omega})-J_{0}^{*}=o_{p}(1)$, where

$$
J_{T}^{*}(\omega)=\frac{1}{4 T}\left[\begin{array}{cc}
Z^{\prime}\left(\Gamma_{A} \otimes I_{T}\right) Z & Z^{\prime}\left(E_{N}^{\prime} P L_{N} \otimes \iota_{T}\right) \\
\left(L_{N}^{\prime} P E_{N} \otimes \iota_{T}^{\prime}\right) Z & 2 L_{N}^{\prime}\left(\Gamma^{-1} \otimes \Gamma^{-1}\right) L_{N}
\end{array}\right]+\frac{1}{T}\left[\begin{array}{cc}
F^{\prime}\left(\Gamma^{-1} \otimes I_{T}\right) F & 0 \\
0 & 0
\end{array}\right]
$$

and both $J_{0}^{*}$ and $J_{T}^{*}(\omega)$ are positive definite.

(ii) $B_{T}^{*}(\hat{\omega})-B_{0}^{*}=o_{p}(1)$, where, in the case of $\bar{m}_{T}^{J}(\hat{\omega}), B_{T}^{*}(\omega)$ can be expressed in (vertically-stacked) "block-row" form, as follows

$$
B_{T}^{*}(\omega)=\left[B_{11 T}^{*}(\omega)^{\prime}, B_{21 T}^{*}(\omega)^{\prime}, B_{22 T}^{*}(\omega)^{\prime}, B_{31 T}^{*}(\omega)^{\prime}, \ldots, B_{N, N-1 T}^{*}(\omega)^{\prime}, B_{N N T}^{*}(\omega)^{\prime}\right]^{\prime}
$$

and the $B_{i j T}^{*}(\omega)$ are ordered by $(i, j)$ according to $s_{N}^{J}$ with

$$
B_{i j T}^{*}(\omega)=\frac{1}{T}\left[\frac{1}{2} \rho_{i j} R_{i j}^{\prime}\left(e_{j}^{\prime} \otimes Z_{j}+e_{i}^{\prime} \otimes Z_{i}\right), R_{i j}^{\prime}\left(e_{i j}^{\prime} \otimes \iota_{T}\right)\right]
$$

We express $B_{T}^{*}(\omega)$ in this way since it then become transparent how it is modified if, for a particular $(i, j), m_{i j, T}(\omega)$ is removed from $m_{T}^{J}(\omega)$. For example, the modification for $\bar{m}_{T}^{C}(\hat{\omega})$ simply removes all $B_{i i T}^{*}(\hat{\omega})$ blocks from $B_{T}^{*}(\omega)$. Using these expressions, and since $T^{-1} \hat{U}^{* \prime} \hat{U}^{*}-\Sigma^{*}=o_{p}(1)$, we obtain the following consistent robust estimator

$$
\hat{V}_{T}^{*(r)}=T^{-1} \hat{A}^{*(r)} \hat{U}^{* \prime} \hat{U}^{*} \hat{A}^{*(r) \prime},
$$

where $\hat{A}^{*(r)}=\left[I_{q},-B_{T}^{*}(\hat{\omega}) J_{T}^{*-1}(\hat{\omega})\right]$. Tests based on 18 will be referred as the robust FQMLE tests and denoted as $\hat{S}_{T}^{*(r)}$. Defining $\hat{W}^{*(r)}=\hat{U}^{*} \hat{A}^{*(r) \prime}$, and noting that $\hat{W}^{*(r) \prime} \iota_{T}=T \bar{m}_{T}(\hat{\omega})$,

$$
\hat{S}_{T}^{*(r)}=\iota_{T}^{\prime} \hat{W}^{*(r)}\left(\hat{W}^{*(r) \prime} \hat{W}^{*(r)}\right)^{-1} \hat{W}^{*(r) \prime} \iota_{T}
$$

\subsection{Tests based on PQMLE}

As discussed in section 2.1. the PQML estimators of the $\theta_{i}$ are $\tilde{\theta}_{i}=\arg \max _{\theta_{i}} \frac{1}{T} \sum_{t=1}^{T} l_{i t}\left(\theta_{i}\right)$, i.e., the consistent univariate GARCH QML estimators, and the constant correlations are estimated as $\tilde{\rho}_{i j}=\frac{1}{T} \sum_{t=1}^{T} \tilde{\zeta}_{i t} \tilde{\zeta}_{j t}^{\prime}$, a function of $\tilde{\theta}$, with $\tilde{\rho}_{i i} \equiv 1$. Therefore, the CCM test indicator $\bar{m}_{T}^{C}(\omega)$, evaluated at $\tilde{\omega}=\left(\tilde{\theta}^{\prime}, \tilde{\rho}^{\prime}\right)^{\prime}$, is constructed from $\left(q_{i j} \times 1\right)$ sub-vectors, with $(i, j)$ ordered according to $s_{N}^{C}$,

$$
\begin{aligned}
\frac{1}{T} \sum_{t=1}^{T}\left(\tilde{\zeta}_{i t} \tilde{\zeta}_{j t}-\tilde{\rho}_{i j}\right) \tilde{r}_{i j, t} & =\frac{1}{T} \sum_{t=1}^{T}\left(\tilde{\zeta}_{i t} \tilde{\zeta}_{j t}-\tilde{\rho}_{i j}\right)\left(\tilde{r}_{i j, t}-\bar{r}_{i j T}(\tilde{\omega})\right) \\
& =\frac{1}{T} \sum_{t=1}^{T}\left(\tilde{\zeta}_{i t} \tilde{\zeta}_{j t}-\rho_{0, i j}\right)\left(\tilde{r}_{i j, t}-\bar{r}_{i j T}(\tilde{\omega})\right),
\end{aligned}
$$


where $\bar{r}_{i j T}(\tilde{\omega}) \equiv \frac{1}{T} \sum_{t=1}^{T} \tilde{r}_{i j, t}$. Note that we retain the notation $\bar{r}_{i j T}(\omega)$ and $\bar{m}_{T}^{C}(\omega)$, for example, since it is consistent with the FQMLE case; however both $\bar{r}_{i j T}(\tilde{\omega})$ and $\bar{m}_{T}^{C}(\tilde{\omega})$ are strictly speaking a function of $\tilde{\theta}$. This formulation considerably simplifies the derivation of the limit distribution of $\sqrt{T} \bar{m}_{T}^{C}(\tilde{\omega})$. In view of this, and also to maintain simplicity in the construction of the various test statistics, we also employ "de-meaned" test variables, $\tilde{r}_{i j, t}-\bar{r}_{i j T}(\tilde{\omega})$, for the FCM test indicator, $\bar{m}_{T}^{J}(\tilde{\omega})$. Thus, the (PQMLE) FCM and CCM test indicators, respectively, are constructed from the following $\left(q_{i j} \times 1\right)$ sub-vectors, with $(i, j)$ ordered according to $s_{N}^{J}$ and $s_{N}^{C}$, respectively,

$$
\begin{aligned}
& \frac{1}{T} \sum_{t=1}^{T}\left(\tilde{\zeta}_{i t} \tilde{\zeta}_{j t}-\tilde{\rho}_{i j}\right)\left(\tilde{r}_{i j, t}-\bar{r}_{i j T}(\tilde{\omega})\right), \quad i \geqslant j \\
& \frac{1}{T} \sum_{t=1}^{T}\left(\tilde{\zeta}_{i t} \tilde{\zeta}_{j t}-\tilde{\rho}_{i j}\right)\left(\tilde{r}_{i j, t}-\bar{r}_{i j T}(\tilde{\omega})\right), \quad i>j
\end{aligned}
$$

However, note that $\frac{1}{T} \sum_{t=1}^{T}\left(\tilde{\zeta}_{i t}^{2}-1\right) \neq 0$, so that $\frac{1}{T} \sum_{t=1}^{T}\left(\tilde{\zeta}_{i t}^{2}-1\right)\left(\tilde{r}_{i i, t}-\bar{r}_{i i T}(\tilde{\omega})\right) \neq \frac{1}{T} \sum_{t=1}^{T}\left(\tilde{\zeta}_{i t}^{2}-1\right) \tilde{r}_{i j, t}$.

Thus, in general, we consider the test indicator $\bar{m}_{T}(\tilde{\omega})=\frac{1}{T} \sum_{t=1}^{T} m_{t}(\tilde{\omega})$ where $m_{t}(\tilde{\omega}),(q \times 1)$, is constructed either from the $\left(q_{i j} \times 1\right)$ sub-vectors 20 , for the FCM test indicator $\bar{m}_{T}^{J}(\tilde{\omega})$, or 21 for the CCM test indicator $\bar{m}_{T}^{C}(\tilde{\omega})$. In order to treat either test indicator, let $\bar{n}_{T}^{\infty}(\omega)=\frac{1}{T} \sum_{t=1}^{T} n_{t}^{\infty}(\omega)$ constructed in the same way from the $\left(q_{i j} \times 1\right)$ sub-vectors

$$
\begin{aligned}
\bar{n}_{i j, T}^{\infty}(\omega) & =\frac{1}{T} \sum_{t=1}^{T}\left(\zeta_{i t}^{\infty} \zeta_{j t}^{\infty}-\rho_{0, i j}\right)\left(r_{i j, t}^{\infty}-\mu_{i j}\left(\omega_{0}\right)\right) \\
& =\frac{1}{T} \sum_{t=1}^{T} n_{i j, t}^{\infty}\left(\omega_{0}\right)
\end{aligned}
$$

where $\mu_{i j}\left(\omega_{0}\right)=E\left[r_{i j, t}^{\infty}\right]_{\omega=\omega_{0}}$ and $\left\|\mu_{i j}\left(\omega_{0}\right)\right\|<\infty$, by Assumption B4 in the Appendix.

The following justifies an asymptotically valid $\chi^{2}$ test statistic, and procedure, based on $\sqrt{T} \bar{m}_{T}(\tilde{\omega})$ :

Proposition 4 Suppose, as described in the Appendix, Assumptions A and B, with B1 and B2, appropriately strengthened for the particular choice of $r_{i j, t}$, hold. Then, $\Sigma=E\left[u_{t}^{\infty}\left(\omega_{0}\right) u_{t}^{\infty}\left(\omega_{0}\right)^{\prime}\right]$ is finite, where $u_{t}^{\infty}(\omega)^{\prime}=\left(m_{t}^{\infty}(\omega)^{\prime}, g_{t}^{\infty}(\theta)^{\prime}\right)$. Furthermore:

(i) $\sqrt{T} \bar{u}_{T}^{\infty}(\omega) \stackrel{d}{\rightarrow} N(0, \Sigma)$, where $\Sigma=E\left[u_{t}^{\infty}\left(\omega_{0}\right) u_{t}^{\infty}\left(\omega_{0}\right)^{\prime}\right]$ is finite; and,

(ii) $\frac{1}{T} \sum_{t=1}^{T} u_{t}(\tilde{\omega}) u_{t}(\tilde{\omega})^{\prime}-\Sigma=o_{p}(1)$, for any $\tilde{\omega}-\omega_{0}=o_{p}(1)$, where $u_{t}(\omega)^{\prime}=\left(m_{t}(\omega)^{\prime}, g_{t}(\theta)^{\prime}\right)$.

Remark 2 Again, for the choice of test variables $r_{i j, t}=\frac{\varepsilon_{i, t-1} \varepsilon_{j, t-1}}{\sqrt{h_{i, t-1}} \sqrt{h_{j, t-1}}}$ we will require $E\left|\varepsilon_{0 i t}\right|^{8}<\infty$. In the case of $\bar{m}_{T}^{J}\left(\omega_{0}\right)$, for example, $\Sigma_{n n} \equiv E\left[n_{t}^{\infty}\left(\omega_{0}\right) n_{t}^{\infty}\left(\omega_{0}\right)^{\prime}\right]$ is block partitioned in a similar way to $\Sigma_{m m}$, given in Remark 1, but with $r_{i j, t}^{\infty}-\mu_{i j}\left(\omega_{0}\right)$ replacing $r_{i j, t}^{\infty}$, throughout. The modification is obvious for $\bar{m}_{T}^{C}\left(\omega_{0}\right)$ which simply removes all $i=j$ entries from $\Sigma_{n n}$.

Some specials cases emerge, however, for either $\bar{m}_{T}^{J}\left(\omega_{0}\right)$ or $\bar{m}_{T}^{C}\left(\omega_{0}\right)$ : 
1. If $r_{t}$ is the same vector of test variables employed for all $i, j$, so that $\mu_{i j} \equiv \mu$, then $\Sigma_{n n}=$ $E\left[v_{0 t} v_{0 t}^{\prime} \otimes \Delta r_{t}\left(\omega_{0}\right) \Delta r_{t}\left(\omega_{0}\right)^{\prime}\right]$, where $\Delta r_{t}\left(\omega_{0}\right)=r_{t}\left(\omega_{0}\right)-\mu\left(\omega_{0}\right)$.

2. If $r_{i j, t}$ is a scalar, let $\Upsilon_{t}=\left\{r_{i j, t}\left(\omega_{0}\right)-\mu_{i j}\left(\omega_{0}\right)\right\},(N \times N)$ and define $\Delta r_{t}\left(\omega_{0}\right)$ to be either $\operatorname{vech}\left(\Upsilon_{t}\right)$, in the case of $\bar{m}_{T}^{J}\left(\omega_{0}\right)$, or $\operatorname{vecl}\left(\Upsilon_{t}\right)$, in the case of $\bar{m}_{T}^{C}\left(\omega_{0}\right)$.

Then $\Sigma_{n n}=E\left[\Delta r_{t}\left(\omega_{0}\right) \Delta r_{t}\left(\omega_{0}\right)^{\prime} \odot v_{0 t} v_{0 t}^{\prime}\right]$.

Proposition 5 Under the assumptions of Proposition 4 , and provided $\Sigma$ is positive definite, $\sqrt{T} \bar{m}_{T}(\tilde{\omega}) \stackrel{d}{\longrightarrow}$ $N(0, V)$, where $V=A_{0} \Sigma A_{0}^{\prime}, A_{0}=\left[I_{q},-B_{0} J_{0}^{-1}\right]$, with $J_{0}=-E\left[\frac{\partial g_{t}^{\infty}\left(\theta_{0}\right)}{\partial \theta^{\prime}}\right], B_{0}=-E\left[\frac{\partial n_{t}^{\infty}\left(\omega_{0}\right)}{\partial \theta^{\prime}}\right]$, and $I_{q}$ is the $(q \times q)$ identity matrix.

In the case of $\bar{m}_{T}^{J}(\tilde{\omega})$, for example, $B_{0}$ is block partitioned as $B_{0}=\left[B_{0, i j}\right]$, with blocks $B_{0, i j}$, stacked (vertically) for $i \geq j, i$ changing faster than $j$, and given by

$$
B_{0, i j}=-E\left\{E\left[\frac{\partial n_{i j, t}^{\infty}\left(\omega_{0}\right)}{\partial \theta^{\prime}} \mid \mathcal{F}_{t-1}\right]\right\}=-E\left[\left(r_{i j, t}^{\infty}-\mu_{i j}\left(\omega_{0}\right)\right) \frac{\partial\left(\zeta_{i t}^{\infty} \zeta_{j t}^{\infty}\right)}{\partial \theta^{\prime}}\right]_{\omega=\omega_{0}}
$$

The modification is obvious for $\bar{m}_{T}^{C}(\tilde{\omega})$ and simply removes all $B_{0, i i}$ blocks from $B_{0}$.

From Proposition 5, and provided $V$ is positive definite, the general form of the test statistic is

$$
\tilde{S}_{T}=T \bar{m}_{T}(\tilde{\omega})^{\prime}\left\{\tilde{V}_{T}\right\}^{-1} \bar{m}_{T}(\tilde{\omega})
$$

which has a $\chi_{q}^{2}$ limiting distribution, under the null, where $\tilde{V}_{T}=V+o_{p}(1)$.

Note that to obtain the right expression for the asymptotic variance estimator of the test indicator one has to use $\left.\operatorname{vech}\left(\tilde{\zeta}_{t} \tilde{\zeta}_{t}^{\prime}-\tilde{\Gamma}\right) \operatorname{or} \operatorname{vecl}\left(\tilde{\zeta}_{t} \tilde{\zeta}_{t}^{\prime}-\tilde{\Gamma}\right)\right), \operatorname{rather}$ than $\operatorname{vech}\left(\tilde{\zeta}_{t} \tilde{\zeta}_{t}^{\prime}\right)$ or $\operatorname{vecl}\left(\tilde{\zeta}_{t} \tilde{\zeta}_{t}^{\prime}\right)$, in the construction of $\bar{m}_{T}^{J}(\tilde{\omega})$ or $\bar{m}_{T}^{C}(\tilde{\omega})$ as given in 20 and 21 , respectively. As earlier, $\tilde{V}_{T}^{(o)}$ and $\tilde{V}_{T}^{(r)}$ will denote OPG and robust variance-covariance matrix estimators, respectively, that we might use for $\tilde{V}_{T}$.

\subsubsection{The OPG-PQMLE Test}

Define the $(T \times q)$ matrix $M \equiv M(\omega)$ to have rows $m_{t}^{\prime}(\omega)$ with the understanding that $\tilde{M} \equiv M(\tilde{\omega})$. Also, let $G=\left(G_{1}, \cdots, G_{N}\right)$ and $G_{\theta}^{*}=\left(G_{1}^{*}, \cdots, G_{N}^{*}\right)$, where the $G_{i} \equiv G\left(\theta_{i}\right)$ and $G_{i}^{*} \equiv G_{i}^{*}(\omega)$ are $\left(T \times K+K^{*}\right)$ matrices, $i=1, \cdots, N$, with rows $\frac{\partial l_{i t}\left(\theta_{i}\right)}{\partial \theta_{i}^{\prime}}$ and $\frac{\partial l_{t}^{*}(\omega)}{\partial \theta_{i}^{\prime}}$, respectively Then, $G$ and $G_{\theta}^{*}$ are $\left(T \times N\left(K+K^{*}\right)\right)$ matrices with rows $g_{t}^{\prime}(\theta)=\left(\frac{\partial l_{1 t}\left(\theta_{1}\right)}{\partial \theta_{1}^{\prime}}, \ldots, \frac{\partial l_{N t}\left(\theta_{N}\right)}{\partial \theta_{N}^{\prime}}\right)$ and $\frac{\partial l_{t}^{*}(\omega)}{\partial \theta^{\prime}}$, respectively. Firstly, and in general, $T^{-1} \tilde{U}^{\prime} \tilde{U}-\Sigma=o_{p}(1)$ by Proposition 4 (ii), where $\tilde{U}=[\tilde{M}, \tilde{G}]$. Secondly, with the additional normality assumption of $\xi_{0 t} \sim N\left(0, I_{N}\right)$, the specification of the log-likelihood for the FQML estimation of parameters is correct. Thus, since $E\left[g_{t}\left(\theta_{0}\right)\right]=0$, the generalized IM equality

\footnotetext{
${ }^{11}$ That is, $G_{i}$ is the matrix having rows univariate GARCH scores, and is a function of $\theta_{i}$, while the rows of $G_{i}^{*}$ contains the FQMLE scores, corresponding to the conditional mean and volatility parameters, $\theta_{i}$, only, but is a function of $\omega$.
} 
implies that

$$
J_{0}=\operatorname{diag}\left(-E\left[\frac{\partial^{2} l_{i t}^{\infty}\left(\theta_{0}\right)}{\partial \theta_{i} \partial \theta_{i}^{\prime}}\right]\right)=\operatorname{diag}\left(E\left[\frac{\partial l_{i t}^{\infty}\left(\theta_{i 0}\right)}{\partial \theta_{i}} \frac{\partial l_{t}^{\infty *}\left(\omega_{0}\right)}{\partial \theta_{i}^{\prime}}\right]\right),
$$

whilst the blocks of $B_{0}$ are

$$
B_{0, i j}=-E\left[\left(r_{i j, t}^{\infty}-\mu_{i j}\left(\omega_{0}\right)\right) \frac{\partial\left(\zeta_{i t}^{\infty} \zeta_{j t}^{\infty}\right)}{\partial \theta^{\prime}}\right]_{\omega=\omega_{0}}=E\left[n_{i j, t}^{\infty}\left(\omega_{0}\right) \frac{\partial l_{t}^{\infty *}\left(\omega_{0}\right)}{\partial \theta^{\prime}}\right]
$$

It then follows that, from Lemma 1 6 and 7, in the Appendix, consistent estimators $J_{0}$ and $B_{0}$ can be obtained as $\operatorname{diag}\left(T^{-1} \tilde{G}_{i}^{\prime} \tilde{G}_{i}^{*}\right)$ and $T^{-1} \tilde{M}^{\prime} \tilde{G}_{\theta}^{*}$, respectively. Therefore, under normality, a consistent estimator for $V=A \Sigma A^{\prime}$ can be obtained as

$$
\tilde{V}_{T}^{(o)}=T^{-1} \tilde{A}^{(o)} \tilde{U}^{\prime} \tilde{U} \tilde{A}^{(o) \prime}
$$

where the matrix $\tilde{A}^{(o)}=\left[I_{q}, \quad-\tilde{M}^{\prime} \tilde{G}_{\theta}^{*} \times \operatorname{diag}\left(\tilde{G}_{i}^{\prime} \tilde{G}_{i}^{*}\right)\right]$. Again, exploiting the fact that $\tilde{G}^{\prime} \iota_{T} \equiv 0$ so that $\tilde{A}^{(o)} \tilde{U}^{\prime} \iota_{T} \equiv \tilde{M}^{\prime} \iota_{T}=T \bar{m}_{T}(\tilde{\omega})$, the test statistic can be expressed in a $T \times R_{u}^{2}$ form, but this time following a regression of $\iota_{T}$ on $\tilde{W}^{(o)}=\tilde{U} \tilde{A}^{(o) \prime}$ :

$$
\tilde{S}_{T}^{(o)}=\iota_{T}^{\prime} \tilde{W}^{(o)}\left(\tilde{W}^{(o) \prime} \tilde{W}^{(o)}\right)^{-1} \tilde{W}^{(o) \prime} \iota_{T}
$$

\subsubsection{The Robust-PQMLE Test}

To construct a robust (to non-normality) test of 22 , first note that $B_{T}(\tilde{\omega})$ (the robust estimator for $B_{0}$ ) can be obtained using the results of Proposition 3 but replacing $\hat{r}_{i j, t}$ by $\tilde{r}_{i j, t}-\bar{r}_{i j T}(\tilde{\omega})$, the demeaned test variables. Thus, if $\widetilde{\bar{R}}_{i j}$ is the $\left(T \times q_{i j}\right)$ matrix having rows $\left(\tilde{r}_{i j, t}-\bar{r}_{i j T}(\tilde{\omega})\right)^{\prime}, t=1, \ldots, T$, then $B_{T}(\tilde{\omega})$ can be expressed in "block form", but with a typical block now being

$$
B_{i j T}(\tilde{\omega})=\frac{1}{2 T} \tilde{\rho}_{i j}^{\prime} \widetilde{R}_{i j}\left(e_{j}^{\prime} \otimes \tilde{Z}_{j}+e_{i}^{\prime} \otimes \tilde{Z}_{i}\right)
$$

ordered by $(i, j)$ according to $s_{N}^{J}$, or $s_{N}^{C}$, for $\bar{m}_{T}^{J}(\tilde{\omega})$, or $\bar{m}_{T}^{C}(\tilde{\omega})$, respectively. In addition, and as a special case of Proposition $3\left(\right.$ i) with $N=1, \rho_{i i}=1,-E\left[\frac{\partial^{2} l_{i t}^{\infty}\left(\theta_{0}\right)}{\partial \theta_{i} \partial \theta_{i}^{\prime}}\right]$ can be consistently estimated by $J_{i T}\left(\tilde{\theta}_{i}\right)=\frac{1}{2 T} \tilde{Z}_{i}^{\prime} \tilde{Z}_{i}+\frac{1}{T} \tilde{F}_{i}^{\prime} \tilde{F}_{i}$, which is positive definite, so that $J_{T}(\tilde{\theta})=\frac{1}{2 T} \tilde{Z}^{\prime} \tilde{Z}+\frac{1}{T} \tilde{F}^{\prime} \tilde{F}$; see, for example, Halunga and Orme (2009, Lemma 1).

Combining the above two results, we obtain the following expression for the robust consistent variance estimator

$$
\tilde{V}_{T}^{(r)}=T^{-1} \tilde{A}^{(r)} \tilde{U}^{\prime} \tilde{U} \tilde{A}^{(r) \prime}
$$

where $\tilde{A}^{(r)}=\left[I_{q},-B_{T}(\tilde{\omega}) J_{T}^{-1}(\tilde{\theta})\right]$. Tests based on this estimator will be referred as the robust PQMLE 
test and will be denoted as $\tilde{S}_{T}^{(r)}$ and can be constructed as

$$
\tilde{S}_{T}^{(r)}=\iota_{T}^{\prime} \tilde{W}^{(r)}\left(\tilde{W}^{(r) \prime} \tilde{W}^{(r)}\right)^{-1} \tilde{W}^{(r) \prime} \iota_{T}
$$

where $\tilde{W}^{(r)}=\tilde{U} \tilde{A}^{(r) \prime}$.

\subsection{Summary}

For each of the FCM and CCM test statistics, and depending on the estimation framework and construction of the variance-covariance matrix, we have a total of eight test statistics, namely,

Table 1: FCM and CCM Test Statistics

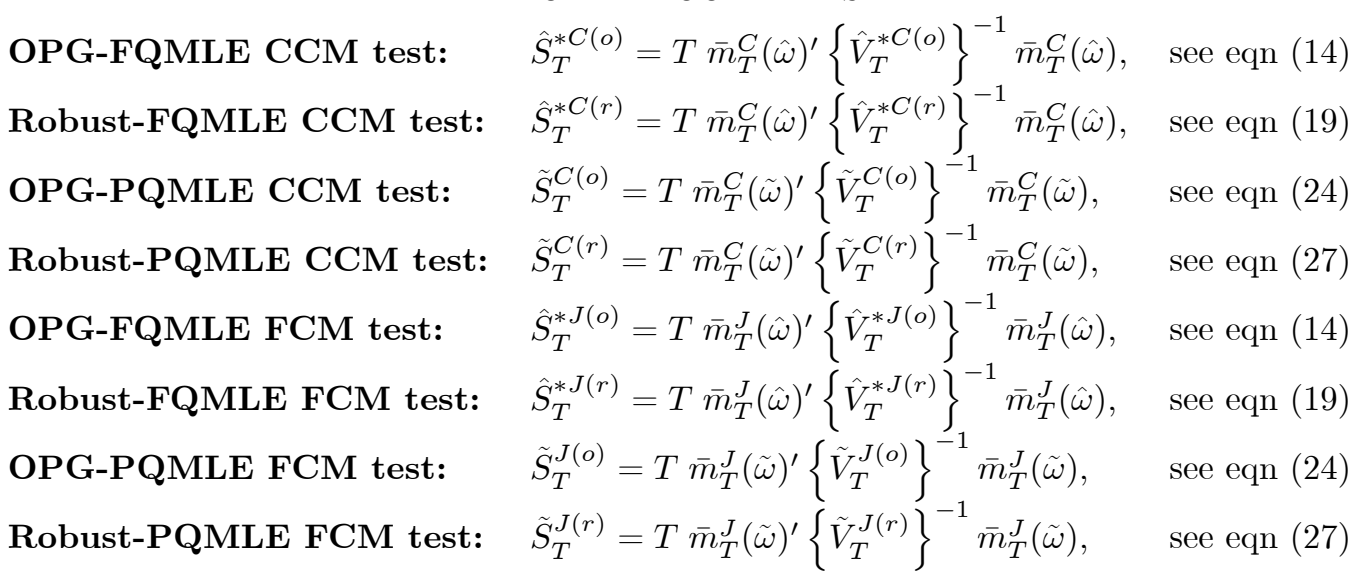

where the hat and tilde represent FQMLE and PQMLE, respectively, the superscript $J$ and $C$ denote FCM and CCM test, respectively, and the superscript $o$ and $r$ signify OPG and robust variance estimator.

\section{OPG and Robust Tse LM Tests}

From 11, it was noted that $\bar{m}_{k l, T}^{L M}(\omega)=\left(\pi_{k l}^{\prime} \otimes I_{q_{k l}}\right) \bar{m}_{(k l) T}^{J}(\omega),\left(q_{k l} \times 1\right)$, where $\bar{m}_{(k l) T}^{J}(\omega)=\frac{1}{T} \sum_{t=1}^{T} v_{t}^{J} \otimes$ $\phi_{k l, t},\left(\frac{q_{k l}}{2} N(N+1) \times 1\right)$, is a joint FCM test indicator vector. Whilst test variables $\phi_{k l, t},\left(q_{k l} \times 1\right)$, are used for the FQMLE case, for the PQMLE case the strategy of using de-meaned test variables, $\phi_{k l, t}-\bar{\phi}_{k l, t}$, is maintained in order to construct a "modified" Tse test.

As a consequence of Proposition 2 it is clear that an asymptotically valid $\chi_{1}^{2}$ Tse statistic designed to test only the $(k, l)^{t h}$ equations for constant correlation, using a vector or test variables $\hat{\phi}_{i j, t}$, can be constructed as

$$
\widehat{L M_{k l, T}}=T \frac{\left(\left(\hat{\pi}_{k l}^{\prime} \otimes I_{q_{i j}}\right) \bar{m}_{(k l) T}^{J}(\hat{\omega})\right)^{2}}{\left(\hat{\pi}_{k l}^{\prime} \otimes I_{q_{i j}}\right) \hat{V}_{(k l)}^{* J}\left(\hat{\pi}_{k l} \otimes I_{q_{k l}}\right)}
$$


where either $\hat{V}_{(k l)}^{* J(o)}$ or $\hat{V}_{(k l)}^{* J(r)}$ can be employed, with the latter providing robustness to non-normality. Similar manipulations can be used to construct joint Tse test statistics, but the following (equivalent) procedures see more straightforward.

Obtain the $\left(T \times q^{C}\right)$ matrix $M \equiv M(\omega)$, to have rows $m_{t}^{L M}(\omega)^{\prime}$, where here $q^{C}=\sum_{k>l} q_{k l}$. That is, $m_{t}^{L M}(\omega)$ is formed by stacking the $\left(q_{k l} \times 1\right)$ sub-vectors $\left(\varepsilon_{k t}^{*} \varepsilon_{l t}^{*}-\rho^{k l}\right) \phi_{k l, t}=\left(\pi_{k l}^{\prime} \otimes I_{q_{k l}}\right)\left(v_{t}^{J} \otimes \phi_{k l, t}\right)$, for the FQMLE case, or $\left(\varepsilon_{k t}^{*} \varepsilon_{l t}^{*}-\rho^{k l}\right)\left(\phi_{k l, t}-\bar{\phi}_{k l, T}\right)=\left(\pi_{k l}^{\prime} \otimes I_{q_{k l}}\right)\left(v_{t}^{J} \otimes\left(\phi_{k l, t}-\bar{\phi}_{k l, T}\right)\right)$, for the PQMLE case, with $(k, l)$ ordered according to $s_{N}^{C}$. Then, with this definition of $M$ and $\bar{m}_{T}^{L M}=\frac{1}{T} \sum_{t=1}^{T} m_{t}^{L M}(\omega)$, the desired test statistic can be constructed as follows (with the first being just Tse's original statistic):

1. The OPG-FQMLE Tse test statistic, $\widehat{L M}_{T}^{*(o)}$.

Construct $\hat{V}_{T}^{* L M(o)}$ using 13 and $\hat{S}_{T}^{*(o)}$ as in 14 giving an equivalent expression to (6) as

$$
\widehat{L M}_{T}^{*(o)}=T \bar{m}_{T}^{L M}(\hat{\omega})^{\prime}\left\{\hat{V}_{T}^{* L M(o)}\right\}^{-1} \bar{m}_{T}^{L M}(\hat{\omega}) .
$$

2. The Robust-FQMLE Tse test statistic, $\widehat{L M}_{T}^{*(r)}$.

Obtain $\hat{A}_{L M}^{*(r)}=\left[I_{q}, \quad-D_{T}^{*}(\hat{\omega}) J_{T}^{*-1}(\hat{\omega})\right]$, where the matrix $D_{T}^{*}(\hat{\omega})$ is constructed by vertically stacking the matrices $D_{k l, T}^{*}(\hat{\omega})=\left(\hat{\pi}_{k l}^{\prime} \otimes I_{q_{k l}}\right) B_{(k l) T}^{*}(\hat{\omega})$, and $B_{(k l) T}^{*}(\hat{\omega})$ is defined in Proposition 3 but where $\hat{R}_{i j}$ is replaced $\hat{R}_{(k l)},\left(T \times q_{k l}\right)$, having rows $\hat{\phi}_{k l, t}^{\prime}$. Then construct $\hat{V}_{T}^{* L M(r)}$ using 18 and $\hat{S}_{T}^{* L M(r)}$ as in 190 giving

$$
\widehat{L M}_{T}^{*(r)}=T \bar{m}_{T}^{L M}(\hat{\omega})^{\prime}\left\{\hat{V}_{T}^{* L M(r)}\right\}^{-1} \bar{m}_{T}^{L M}(\hat{\omega}) .
$$

3. The OPG-PQMLE Tse test statistic, $\widetilde{L M}_{T}^{(o)}$.

Construct $\tilde{V}_{T}^{L M(o)}$ using 23 and $\tilde{S}_{T}^{(o)}$ as in 24 giving

$$
\widetilde{L M}_{T}^{(o)}=T \bar{m}_{T}^{L M}(\tilde{\omega})^{\prime}\left\{\tilde{V}_{T}^{L M(o)}\right\}^{-1} \bar{m}_{T}^{L M}(\tilde{\omega})
$$

4. The Robust-FQMLE Tse test statistic, $\widetilde{L M}_{T}^{(r)}$.

Obtain $\tilde{A}_{L M}^{(r)}=\left[I_{q}, \quad-D_{T}(\tilde{\omega}) J_{T}^{-1}(\tilde{\theta})\right]$, where the matrix $D_{T}(\tilde{\omega})$ is constructed by vertically stacking the matrices $D_{k l, T}(\tilde{\omega})=\left(\pi_{k l}^{\prime} \otimes I_{q_{k l}}\right) B_{(k l) T}(\tilde{\omega})$, and $B_{(k l) T}(\tilde{\omega})$ is defined by 25), but where $\widetilde{\bar{R}}_{i j}$ is replaced by $\widetilde{\bar{R}}_{(k l)},\left(T \times q_{k l}\right)$, having rows $\left(\tilde{\phi}_{k l, t}-\widetilde{\bar{\phi}}_{k l, T}\right)$. Then construct $\tilde{V}_{T}^{L M(r)}$ using 26. and $\hat{S}_{T}^{* L M(r)}$ as in 27) giving

$$
\widetilde{L M}_{T}^{(r)}=T \bar{m}_{T}^{L M}(\tilde{\omega})^{\prime}\left\{\tilde{V}_{T}^{L M(r)}\right\}^{-1} \bar{m}_{T}^{L M}(\tilde{\omega}) .
$$

The above derivations also make it transparent how to construct a joint Tse test of a subset of the 
constant conditional correlations, rather than for all $\frac{1}{2} N(N-1)$. However, if all $\frac{1}{2} N(N-1)$ constant conditional correlations are to be tested, the derivations in the proof of Proposition 3 (see Shadat and Orme, 2015) imply that $D_{T}^{*}(\omega)$ and $D_{T}(\omega)$ can expressed as

$$
\begin{aligned}
D_{T}^{*}(\omega) & =\frac{1}{4 T}\left[\Phi^{\prime}\left(L_{N}^{\prime} P E_{N} \otimes I_{T}\right) Z, 2 \Phi^{\prime}\left(L_{N}^{\prime}\left(\Gamma^{-1} \otimes \Gamma^{-1}\right) L_{N} \otimes \iota_{T}\right)\right] \\
D_{T}(\omega) & =\frac{1}{4 T}\left[\bar{\Phi}^{\prime}\left(L_{N}^{\prime} P E_{N} \otimes I_{T}\right) Z\right]
\end{aligned}
$$

where $\Phi=\operatorname{diag}\left(\Phi_{k l}\right),\left(\frac{T}{2} N(N-1) \times q^{C}\right)$ with $\Phi_{k l},\left(T \times q_{k l}\right)$ having rows $\phi_{k l, t}, t=1, \ldots, T$, whilst $\bar{\Phi}=\operatorname{diag}\left(\bar{\Phi}_{k l}\right),\left(\frac{T}{2} N(N-1) \times q^{C}\right)$ with $\bar{\Phi}_{k l},\left(T \times q_{k l}\right)$ having rows $\phi_{k l, t}-\bar{\phi}_{k l, t}, t=1, \ldots, T$.

\section{Monte Carlo Evidence}

In this section, we present Monte Carlo evidence on the finite sample behaviour of the 12 , for both FQMLE and PQMLE procedures, for $N=5$ equations: the $8 \mathrm{CM}$ tests described in Table 1 and the 4 Tse "LM" tests described in (28)-31). The parameter values for the null and alternative Data Generating Processes (DGPs), where possible, are taken from the existing literature (e.g., Engle and Ng (1993), Tse (2000), Lundbergh and Teräsvirta (2002), Halunga and Orme (2009)). For each experiment, two series of 1200 and 700 data realizations were generated with the first 200 observations being discarded to avoid initialization effects, yielding sample sizes of $T=1000$ and 500, respectively. Each model is replicated and estimated, 10,000 times (to obtain empirical significance levels) and 2000 times (for robustness to non-normality and power experiments).

In practice, however, there is likely to be considerable uncertainty about the precise form of misspecification in the MGARCH CCC structure and so that any "selected" alternative may often be misspecified, leading to an "incorrect" set of test variables. Thus, the primary purpose of the Monte Carlo study, here, is to compare the finite sample performance (empirical significance levels, robustness and power) of the various tests, each constructed with a common set of test variables, in order to see if a ranking emerges. Following Tse (2000), the common scalar test variable employed for this purpose for is $r_{i j, t}(\omega)=\zeta_{i, t-1} \zeta_{j, t-1}$, although in a demeaned form following PQMLE ${ }^{12}$ All simulation experiments are conducted in GAUSS programming language.

\footnotetext{
${ }^{12}$ Although not reported here, simulations were also carried out using $\zeta_{i, t-2} \zeta_{j, t-2}$ as test variables yielding qualitatively similar results. These are available from the authors upon request.
} 


\subsection{Empirical Significance Levels}

We employ AR(1)-CCC-GARCH $(1,1)$ DGP for $N=5$ as our null model; viz.,

$$
\begin{aligned}
y_{i t} & =\varphi_{i 0}+\varphi_{i 1} y_{i, t-1}+\varepsilon_{i t}, \quad i=1, \cdots, 5 \\
\operatorname{Var}\left(\varepsilon_{t} \mid \mathcal{F}_{t-1}\right) & =H_{t} \Rightarrow E\left[\varepsilon_{i t}^{2} \mid \mathcal{F}_{t-1}\right]=h_{i t}, \quad \varepsilon_{t}=H_{t}^{1 / 2}(\omega) \xi_{t}, \xi_{t} \sim N(0, I) \\
h_{i, t} & =\alpha_{i 0}+\alpha_{i 1} \varepsilon_{i, t-1}^{2}+\beta_{i 1} h_{i, t-1}, \\
H_{t} & =D_{t} \Gamma D_{t}, D_{t}=\operatorname{diag}\left(\sqrt{h_{i t}}\right) \text { and } \\
\Gamma & =\left\{\rho_{i j}\right\}, i, j=1, \cdots, 5 \text { with } \rho_{i i}=1 .
\end{aligned}
$$

Three experiments are considered E1, E2 and E3 (and the true parameter vectors employed are given in Table A1 of Shadat and Orme (2015)). These provide models with relatively low (ranging between 0.20 and 0.37 ), mixed (ranging between 0.30 and 0.80 ) and high (ranging between 0.62 and 0.80) correlation structure, respectively, for $\Gamma$. For all three DGPs the same true parameter values for $\varphi_{i}^{\prime}$ and $\eta_{i}^{\prime}=\left(\alpha_{i 0}, \alpha_{i 1}, \beta_{i 1}\right)$ are used. Note that the experiments considered various degrees of volatility persistence; however, to save space, we report the results only for $\alpha_{1}+\beta_{1}=0.85$ since the results are qualitatively similar in other cases. Tse (2000) also reports "correlations seem to play a role in determining the rate of convergence to the nominal size. Models with low correlations are less subject to over-rejection in small samples.... the persistence of the conditional variance does not have much effect" (p. 115).

Table 2 reports the rejection frequencies when the null of the CCC is true under both Gaussian and non-Gaussian errors. Apart from investigating the robustness of these tests under non-normality, where the elements of $\xi_{0 t}$ are iid as $t(6)$, this also offers some evidence on the robustness of the procedure to violations of the underlying moment assumptions, since for this choice of test variables $8^{\text {th }}$ order moments are required. The results are reported for a nominal significance level of $5 \%$.

First, under Gaussian errors the original Tse test and other OPG-FQMLE type tests $\left(\widehat{L M}_{T}^{*(o)}, \hat{S}_{T}^{* C(o)}\right.$ and $\hat{S}_{T}^{* J(o)}$ ) tend to over-reject, for all DGPs, even with $T=1000$ (particularly $\hat{S}_{T}^{* J(o)}$ ). The robust versions $\left(\widehat{L M}_{T}^{*(r)}, \hat{S}_{T}^{* C(r)}\right.$ and $\left.\hat{S}_{T}^{* J(r)}\right)$ are much superior. Interestingly, the OPG-PQMLE tests $\left(\widetilde{L M}_{T}^{(o)}\right.$, $\tilde{S}_{T}^{C(o)}$ and $\tilde{S}_{T}^{J(o)}$ ) perform better than the corresponding OPG-FQMLE tests, although, $\tilde{S}_{T}^{C(o)}$ and $\tilde{S}_{T}^{J(o)}$ are still oversized. However, the empirical significance levels of their robust counterparts, both FQMLE and PQMLE and including $\widehat{L M}_{T}^{*(r)}$ and $\widetilde{L M}_{T}^{(r)}$, are reasonably close to the nominal size of $5 \%$, even when $T=500$. Second, in the case of experiments with mixed and high correlation structure (E2 and E3), the size distortions of OPG-FQMLE tests are relatively higher compared to the low correlation structure whilst the robust version of these statistics appears to correct this size distortion. On the other hand, the rejection rates for OPG-PQMLE tests under low correlation structure (E1), in particular for $\tilde{S}_{T}^{C(o)}$ 
and $\tilde{S}_{T}^{J(o)}$, are higher than for E2 and E3, although their robust version again corrects this deformity. The finding from these experiments that size performance depends on correlation is in line with that of Tse (2000) where the Monte Carlo experiments were performed for $N=2$. Third, with $\xi_{0 t} \sim t(6)$ all OPG-FQMLE tests $\left(\widehat{L M}_{T}^{*(o)}, \hat{S}_{T}^{* C(o)}\right.$ and $\left.\hat{S}_{T}^{* J(o)}\right)$ over-reject under all correlation structures, but this distortion is more severe in high and mixed correlation models. In particular, Tse's original LM test $\left(\widehat{L M}_{T}^{*(o)}\right)$ is very sensitive to departures from normality. The robust-FQMLE version of all tests reduces the over-rejection rate substantially. The empirical significance levels of the robust versions of Tse's test (particularly $\widetilde{L M}_{T}^{(r)}$, as for the case with normal errors) and the robust CCM tests $\left(\hat{S}_{T}^{* C(r)}\right.$ and $\left.\tilde{S}_{T}^{C(r)}\right)$, in general, are close to nominal level of $5 \%$ while the robust FCM tests $\left(\hat{S}_{T}^{* J(r)}\right.$ and $\left.\tilde{S}_{T}^{J(r)}\right)$ can over or under-reject.

In summary: the OPG-FCM tests over-reject; all test statistics perform better in low correlation experiments; in general, the robust versions of tests perform better than the OPG; and, in particular,Tse's modified robust PQMLE test and the robust CCM PQMLE tests (i.e., $\widetilde{L M}(r)$ and $\tilde{S}_{T}^{C(r)}$ ) provide quite reliable significance levels. All robust tests provide significant size correction under non-normal errors.

\subsection{Robustness to Misspecified Univariate Volatility}

In total,we consider 12 experiments (M1a-M1c, M2a-M2c, M3a-M3c and M4a-M4c), each within the regression context to investigate, via Monte Carlo simulation, the impact of violations in the univariate GARCH specification, but when the true correlation structure for $\zeta_{0 t}$ is constant with Gaussian error. The conditional mean parameters and the correlation structures remain the same as those previously employed, as detailed in Table A1 of Shadat and Orme (2015). For M1, M2 and M3 the univariate volatility specifications of all five variables are governed by the GJR, higher order GARCH (i.e., $\operatorname{GARCH}(2,2)$ ) and the EGARCH models, respectively whereas for M4 all 5 variables are subject to volatility spillover via an ECCC model. The suffix a, b or $\mathrm{c}$ associated with these experiments indicate low, mixed and high correlation structure, respectively, for $\Gamma$. Specifically, we employ the following DGPs, for $i=1, \ldots, 5$ :

1. M1 (GJR): $h_{i t}=a_{i 0}+b_{i 1}\left[\left|\varepsilon_{i t-1}\right|-b_{i 2} \varepsilon_{i t-1}\right]^{2}+b_{i 3} h_{i t-1}$, with parameter vectors, for each $i,(0.005,0.23,0.23,0.70),(0.005,0.30,0.17,0.70)$, $(0.005,0.25,0.20,0.70),(0.005,0.28,0.15,0.70)$, and $(0.005,0.20,0.23,0.70)$.

2. $\mathrm{M} 2(\operatorname{GARCH}(2,2)): h_{i t}=a_{i 0}+a_{i 1} \varepsilon_{i, t-1}^{2}+a_{i 2} \varepsilon_{i, t-2}^{2}+b_{i 1} h_{i, t-1}+b_{i 2} h_{i, t-2}$, with parameter vectors $(0.01,0.15,0.05,0.60,0.15),(0.02,0.25,0.05,0.50,0.15)$, $(0.15,0.10,0.05,0.70,0.10),(0.05,0.10,0.01,0.70,0.14)$, and $(0.05,0.20,0.05,0.65,0.05)$.

3. M3 (EGARCH): $\log \left(h_{i, t}\right)=a_{i 0}+b_{i 1} \log \left(h_{i, t-1}\right)+b_{i 2}\left[\left|\zeta_{i t-1}\right|-b_{i 3} \zeta_{i t-1}\right]$, with parameter vectors, for each $i,(-0.23,0.90,0.25,0.30),(-0.20,0.70,0.25,0.20)$, $(0.23,0.60,0.25,0.20),(0.20,0.80,0.28,0.15)$, and $(-0.30,0.90,0.40,0.15)$. 
4. M4 (ECCC): $h_{i t}=\alpha_{i 0}+\alpha_{i 1} \varepsilon_{i, t-1}^{2}+\beta_{i i} h_{i, t-1}+\sum_{j \neq i} \beta_{i j} h_{j, t-1}$,

with spillover parameter vectors, for each $i,(0.01,0.02,0.015,0.03),(0.02,0.06,0.03,0.04)$, $(0.03,0.01,0.025,0.015),(0.05,0.03,0.02,0.01)$, and $(0.002,0.035,0.04,0.02)$.

The GARCH parameters for the ECCC model remain the same as AR(1)-CCC-GARCH $(1,1)$ null model.

Although EGARCH and ECCC models are not formally within GARCH family of alternatives, as the other DGPs considered here, they represent alternative misspecifications of volatility not captured by GJR and GARCH(2,2). In order to conserve space, we report in Table 3 only the results for experiments M1 (GJR) and M3 (EGARCH), since the results for M2 $(\operatorname{GARCH}(2,2))$ and M4 (ECCC) are qualitatively similar to M1 (GJR), but summarise the main findings for all experiments. Full results are provided in Shadat and Orme (2015). Rejection frequencies are based on both the $5 \%$ empirical and nominal critical values (with the latter in the parenthesis) and with 2000 replications where the data are generated with normal errors; i.e., in the former case, and for each test procedure, "sizeadjusted" rejection frequencies are reported, calculated using the empirical critical value that delivers a 5\% significance level for the simulations reported in Section 5.1. All robust tests and PQMLE-OPG tests are relatively insensitive to GJR, $\operatorname{GARCH}(2,2)$ and ECCC volatility spillover DGPs and for all correlation structures; except joint tests $\tilde{S}_{T}^{* J(r)}$ and $\tilde{S}_{T}^{* J(o)}$ under the $\operatorname{GARCH}(2,2)$ DGP. On the other

hand, the FQMLE-OPG versions, particularly $\hat{S}_{T}^{* J(o)}$, over-reject the null of CCC and the over-rejection is substantially higher when we use the nominal significance level. Since the FCM test indicator entails the volatility moment condition, these tests display some power when this moment condition is violated. In case of the EGARCH alternative, all tests, although to a much lesser extent $\widehat{L M}_{T}^{*(r)}$ and $\widetilde{L M}_{T}^{(r)}$, are quite sensitive to the volatility misspecfications embodied in M3b and M3c (i.e., with mixed and high correlation). In these cases, all tests over-reject significantly the null of CCC and the rejection rates are similar for both empirical and nominal significance level. However, for M3a (low correlation), all the robust tests are less sensitive to univariate conditional variance misspecification.

\subsection{Power Results}

To examine power, we consider three types of MGARCH models with time varying correlations. The $\operatorname{AR}(1)$ conditional mean specification, and parameters, remain as in 32 but now we examine three alternative specifications for the conditional variance matrix $H_{t}=\operatorname{Var}\left(\varepsilon_{t} \mid \mathcal{F}_{t-1}\right)$. The first is Engle's (2002) DCC-GARCH(1,1) model where the dynamic correlation matrix, $\Gamma_{t}$, is given as

$$
\begin{aligned}
\Gamma_{t} & =\left(I \odot \Psi_{t}\right)^{-1 / 2} \Psi_{t}\left(I \odot \Psi_{t}\right)^{-1 / 2}=\operatorname{diag}\left(\Psi_{t}\right)^{-1 / 2} \Psi_{t} \operatorname{diag}\left(\Psi_{t}\right)^{-1 / 2}, \\
\Psi_{t} & =(1-\tilde{\alpha}-\tilde{\beta}) \bar{\Gamma}+\widetilde{\alpha} \zeta_{t-1} \zeta_{t-1}^{\prime}+\widetilde{\beta} \Psi_{t-1},
\end{aligned}
$$


where $\tilde{\alpha}$ and $\tilde{\beta}$ are nonnegative scalar parameters and $\alpha+\beta<1$ and $\bar{\Gamma}$ is constant (time invariant) $5 \times 5$ symmetric positive definite matrix, with ones on the diagonal. Secondly, we consider the following Varying Correlation (VC) model of Tse and Tsui (2002)

$$
\Gamma_{t}=(1-a-b) \bar{\Gamma}+a \Gamma_{t-1}+b \Psi_{t-1},
$$

where $a$ and $b$ are nonnegative scalar parameters, satisfying $a+b \leq 1$, and $\Psi_{t-1}$ is the $5 \times 5$ sample correlation matrix of $\left\{\zeta_{t-1}, \cdots, \zeta_{t-5}\right\}$ and its $(i, j)^{t h}$ element is given by:

$$
\psi_{i j, t-1}=\frac{\sum_{m=1}^{5} \zeta_{i, t-m} \zeta_{j, t-m}}{\left(\sum_{m=1}^{5} \zeta_{i, t-m}^{2}\right)^{1 / 2}\left(\sum_{m=1}^{5} \zeta_{j, t-m}^{2}\right)^{1 / 2}} .
$$

Finally we consider the BEKK model of Engle and Kroner (1995),

$$
H_{t}=C_{B}+A_{B}^{\prime}\left(\varepsilon_{t-1} \varepsilon_{t-1}^{\prime}\right) A_{B}+B_{B}^{\prime} H_{t-1} B_{B} .
$$

In the following experiments the diagonal BEKK (DBEKK) model is employed where the parameter matrices $A_{B}$ and $B_{B}$ are $5 \times 5$ diagonal matrices.

Seven experiments are considered: P1, P2 and P3 follow the DCC DGP (33), P4 and P5 follow VC DGP (34) and remaining two, P6 and P7, follow the DBEKK DGP 35. In all cases, the individual volatility specification for all variables is retained from earlier size experiment, whilst for the DCC and VC DGPs the constant $\bar{\Gamma}$ matrix is set to the previously defined mixed correlation structure (see Section 5.1). The remaining true parameter vectors are given in Shadat and Orme (2015).

Again, to conserve space, we only report detailed results for the DCC DGP, P1-P3, but summarise the main findings for all experiments. Full results are provided in Shadat and Orme (2015). Table 4 presents the size-adjusted power (and nominal) results with 2000 replications, based on a $5 \%$ empirical (respectively nominal) critical values and the data are generated assuming normality. As a measure of the variability of the conditional correlation coefficients, in experiments $\mathrm{P} 1$ to $\mathrm{P} 7$, we also report in Shadat and Orme (2015) the average, maximum and minimum values of the true conditional correlation coefficients across the 2000 Monte Carlo replications of each $T=1000$ sample.

When the true DGP is the DCC, P3 has the largest variability in correlations followed by P2 and P1; i.e., variability increases as $\widetilde{\alpha}$ increases and $\widetilde{\beta}$ decreases. In general, the FCM tests are found to have higher power in all three DCC experiments. However, as the variability in correlation decreases power decreases. The Tse and CCM tests also exhibit good power properties: even with $T=500$, and all tests have high power especially for the P2 and P3 DGPs. In case of the VC and BEKK DGPs the conclusions are quite similar. P5 and P7 have larger variability in correlations than $\mathrm{P} 4$ and $\mathrm{P} 6$, respectively, and the performance of all the tests reflect that. 
Although the OPG-FQMLE tests exhibit higher nominal power, in terms of the size-adjusted power the robust-FQMLE and robust-PQMLE versions of these do not cost much in this respect, especially in view of the lack of robustness to non-normality of the former.

\section{Concluding Remarks}

In this paper, we have considered a set of asymptotically valid Conditional Moment (CM) tests designed to assess a constant correlation assumption and/or the individual GARCH specifications in a MGARCH model. In particular, we consider both the FQMLE and PQMLE framework for the CCC model, noting that there is very little in the existing literature for the latter case. These tests are very easy to implement and include OPG versions - a popular variant in the applied literature but whose asymptotic validity is based on an assumption of normality - and non-normality robust versions. In so doing, we also provide a simple expression for a consistent estimator for the hessian in the FQMLE framework. Our approach accommodates Tse's (2000) LM test, originally proposed as a OPG-FQMLE type test, so that we are able to provide the PQMLE and robust version of this popular test, as well.

We examine the finite sample performance of these asymptotically valid tests via a small Monte Carlo study, with $N=5$ time series rather than the usual bivariate model, which indicates that, in general, all tests have empirical significance levels that are reasonably close to the nominal value of $5 \%$ but that the robust versions are slightly preferred, even under normality. It also appears that, under the null, whilst the degree of univariate volatility persistence has little detrimental effect, low correlation is associated with better empirical significance levels. As anticipated, though, under non-normality (but otherwise correct model specification), the robust version of any particular test exhibits far superior finite sample behaviour relative to its OPG variant with all OPG-FQMLE tests over-rejecting. Interestingly, the OPG-PQMLE based tests exhibit more robustness than the corresponding OPG-FQMLE tests. When the GARCH error assumption of the null model is violated by introducing a volatility spillover effect the Monte Carlo evidence suggests that there is little impact on empirical significance levels. When there is no volatility spillover but simply one GARCH equation misspecified, and a high correlation structure, all tests experience increased empirical rejection rates. This is especially true in the case of the EGARCH alternative with the FCM tests (which test jointly the individual volatility specifications and the CCC assumption) being most sensitive, as one might expect. However, an important result that emerges for applied workers is that although Tse's original FQMLE test is also affected, as it employs all the indicators of the FCM tests, the modified and robust PQMLE version developed in this paper appears to be much less sensitive to univariate volatility misspecification.

Turning to power, which depends on the variability of the true correlation parameter, it is found that Tse's test and FCM tests have good power, with the former being slightly more powerful, even in models 
with less dispersed correlations. Furthermore, for both the Tse and FCM tests, there is comparability across both FQMLE and PQMLE frameworks. Disappointingly, the CCM tests (designed only to assess the CCC assumption) show comparatively lower power - particularly in models with less dispersed correlations.

In conclusion, when testing the assumption CCC there appears to be little difference between the FQMLE and PQMLE approach and, in both cases, non-normality robust versions of the tests exhibit reasonable finite sample behaviour under the null. However, within the panoply of procedures considered and with a common choice of test variable, the robust version of the Tse's test, both for the FQMLE and PQMLE, has very good empirical significance level and power properties and would appear to recommend itself, although it is not entirely robust, in general, to misspecified volatility.

\section{Appendix: Assumptions and Proofs}

Unless stated otherwise all definitions are as in the main text, the Euclidean norm of a matrix $A$ is denoted $\|A\|=\sqrt{\operatorname{tr}\left(A^{\prime} A\right)}$, and the properties of $h_{i t}$ and $h_{i t}^{\infty}$, as discussed in Halunga and Orme (2009, Appendix), are exploited. Whilst only briefly discussed in this Appendix, exhaustive proofs of all results are provided (freely on-line) in Shadat and Orme (2015).

Write $w_{i t}^{\prime} \varphi_{i}=\varphi_{i 1}(L) y_{i t}+d_{i t}^{\prime} \varphi_{i 2}$ and $h_{i t}=\alpha_{i 0}+A_{i}(L) \varepsilon_{i t}^{2}+B_{i}(L) h_{i t}=a_{i t}+B_{i}(L) h_{i t}$, where $a_{i t}=\alpha_{i 0}+A_{i}(L) \varepsilon_{i t}^{2}=\alpha_{i 0}+\sum_{k=1}^{q} \alpha_{i k} \varepsilon_{i, t-k}^{2}$. As employed, for example, in Ling and McAleer (2003), Berkes, Horváth and Kokoszka (2003) and Halunga and Orme (2009), the following assumptions ensure the identifiability, stationarity and ergodicity of the above process 13

\section{Assumptions A}

A1 The parameter space, $\Theta$, is compact and $\omega_{0}$ lies in the interior of $\Theta$.

A2 The elements of $d_{i t}^{\prime}$ are strictly stationary and ergodic and all roots of $1-\varphi_{i 1}(L)=1-\varphi_{i 11} L-$ $\phi_{i 12} L^{2}-\ldots-\phi_{i 1 p} L^{p}=0, \phi_{i 1 p} \neq 0, p$ known, lie outside the unit circle, for all $i$.

A3 (i) All the roots of $1-A_{i}(z)-B_{i}(z)=0$ lie outside the unit circle.

(ii) The parameter space is constrained such that $0<\lambda \leq \min _{i, l}\left\{\eta_{i l}\right\} \leq \max _{i, l}\left\{\eta_{i l}\right\}<\Lambda$, $l=1, \ldots, p+q+1$, where $\lambda$ and $\Lambda$ are independent of $\omega$.

(iii) The polynomials $A_{i}(z)$ and $1-B_{i}(z)$ are coprimes.

Ling and McAleer (2003), for example, required that $E\left(\varepsilon_{0 t}^{6}\right)<\infty$ to ensure asymptotic normality of the QML estimator in the ARMA-GARCH model. This is also sufficient here, but with additional moment restrictions on $d_{i t}$ and the test variables $r_{i j, t}$, as follows:

\section{Assumptions B}

B1 $E\left|\varepsilon_{0 i t}\right|^{6}<\infty$ for all $i, t$.

B2 $E\left[\left\|d_{i t}\right\|^{6}\right]<\infty$, for all $i, t$.

B3 $\sum_{t=1}^{T} E \sup _{\omega}\left|\varepsilon_{i t} \varepsilon_{j t}\right|^{l}\left\|r_{i j, t}^{\infty}-r_{i j, t}\right\|=O(1)$, at most, for all $i, j, t$ and $l=0,1$.

B4 $E \sup _{\omega}\left|\varepsilon_{i t} \varepsilon_{j t}\right|^{l}\left\|r_{i j, t}^{\infty}\right\|^{2}<\infty$ for all $i, j, t$, and $l=0,1,2$.

\footnotetext{
${ }^{13}$ As discussed by Nelson and Cao (1992), although sufficient, A3(ii) is not necessary to ensure non-negative conditional variances.
} 
B5 $E \sup _{\omega}\left|\varepsilon_{i t} \varepsilon_{j t}\right|^{l}\left\|\frac{\partial r_{i j, t}^{\infty}}{\partial \omega}\right\|<\infty$, at most, for all $i, j, t$ and $l=0,1$.

Remark 3 (i) A1, A2, B1 and B2 imply that $E \sup _{\omega}\left|\varepsilon_{i t}\right|^{6}<\infty$ uniformly in $i, t$, where $\varepsilon_{i t}=\varepsilon_{0 i t}-$ $w_{i t}^{\prime}\left(\varphi_{i}-\varphi_{i 0}\right)$, and also that $E\left|y_{i t}\right|^{6}<\infty$ for all $i, t$, so that $E\left[\left\|w_{i t}\right\|^{6}\right]<\infty$, for all $i, t$. (ii) Extensions of Halunga and Orme (2009, Proposition 4) imply that B3-B5 also hold with $z_{i t}$ replacing $r_{i j, t}$. (iii) Assumptions A, B1 and B2 are sufficient to establish the consistency and asymptotic normality of both the FQMLE and PQMLE, and the consistency of variance estimators based on an OPG formulation. (iv) Depending on the choice of $r_{i j, t}$, B1 and B2 may need strengthening, in view of the demands of B4, in order to establish both the asymptotic normality of our test indicators and the consistency of the various asymptotic variance estimators employed in constructing the $\chi^{2}$ test statistics.

Case 1 For $r_{i j, t}=\frac{\varepsilon_{i, t-1} \varepsilon_{j, t-1}}{\sqrt{h_{i, t-1}} \sqrt{h_{j, t-1}}}$, B3-B5 hold provided B1 and B2 are replaced by

$B 1^{*} E\left|\varepsilon_{0 i t}\right|^{8}<\infty$ for all $i, t$.

$B 2^{*} E\left[\left\|d_{i t}\right\|^{8}\right]<\infty$ for all $i, t$.

Proof. This follows from similar arguments to those employed by Halunga and Orme (2009).

We first establish some preliminary results that will be of use later.

Lemma 1 Let $\left\{x_{t}\right\}_{t=1}^{T}$ be a sample of stationary ergodic random variables, such that the random vector functions $w_{t}(\omega) \equiv w\left(x_{t} ; \omega\right)$ and $z_{t}(\omega) \equiv z\left(x_{t} ; \omega\right), t=1, \ldots, T$, satisfy $\frac{1}{\sqrt{T}} \sum_{t=1}^{T} \sup _{\omega}\left\|w_{t}(\omega)-z_{t}(\omega)\right\|=$ $o_{p}(1)$.

(i) Then

$$
\sup _{\omega}\left\|\frac{1}{\sqrt{T}} \sum_{t=1}^{T} w_{t}(\omega)-\frac{1}{\sqrt{T}} \sum_{t=1}^{T} z_{t}(\omega)\right\|=o_{p}(1) .
$$

(ii) If $\operatorname{Esup}_{\omega}\|w(x ; \omega)\|^{2}<\infty$, where $\omega \in \Omega$ a compact set, then (in addition)

$$
\sup _{\omega}\left\|\frac{1}{T} \sum_{t=1}^{T} w_{t}(\omega) w_{t}(\omega)^{\prime}-\frac{1}{T} \sum_{t=1}^{T} z_{t}(\omega) z_{t}(\omega)^{\prime}\right\|=o_{p}(1) .
$$

Proof. Follows from the properties of sup, the triangle inequality and Cauchy-Schwartz.

Remark 4 Under the conditions of Lemma 1, E $\sup _{\omega}\|w(x ; \omega)\|^{2}<\infty$ so that $E\left[w_{t}\left(\omega_{0}\right) w_{t}\left(\omega_{0}\right)^{\prime}\right]$ is finite. Then, by a Uniform Law of Large Numbers and the triangle inequality, for any $\hat{\omega}-\omega_{0}=o_{p}(1)$, $\frac{1}{T} \sum_{t=1}^{T} z_{t}(\hat{\omega}) z_{t}(\hat{\omega})^{\prime}-E\left[w_{t}\left(\omega_{0}\right) w_{t}\left(\omega_{0}\right)^{\prime}\right]=o_{p}(1)$.

Proposition 6 Under Assumptions $A$ and B1, B2:

(i) $E \sup _{\omega}\left\|g_{t}^{\infty *}(\omega)\right\|^{2}<\infty$;

(ii) $\frac{1}{\sqrt{T}} \sum_{t=1}^{T} \sup _{\omega}\left\|g_{t}^{\infty *}(\omega)-g_{t}^{*}(\omega)\right\|=o_{p}(1)$.

In addition, and adding $B 3$ and $B_{4}$ :

(iii) $E \sup _{\omega}\left\|m_{t}^{\infty}(\omega)\right\|^{2}<\infty$;

(iv) $\frac{1}{\sqrt{T}} \sum_{t=1}^{T} \sup _{\omega}\left\|m_{t}^{\infty}(\omega)-m_{t}(\omega)\right\|=o_{p}(1)$. 
Proof.

(i) The scores are

$$
\begin{aligned}
\partial l_{t}^{\infty *}(\omega) / \partial \theta_{i} & =f_{i t}^{\infty} \varepsilon_{i t}^{\infty *}+\frac{1}{2}\left(\zeta_{i t}^{\infty} \varepsilon_{i t}^{\infty *}-1\right) z_{i t}^{\infty}, \\
\partial l_{t}^{\infty *}(\omega) / \partial \rho_{i j} & =\varepsilon_{i t}^{\infty *} \varepsilon_{j t}^{\infty *}-\rho^{i j}, \quad i>j .
\end{aligned}
$$

The result follows from the arguments employed by Halunga and Orme (2009).

(ii) From 36 ,

$$
\begin{aligned}
\frac{1}{\sqrt{T}} \sum_{t=1}^{T} \sup _{\omega}\left\|\frac{\partial l_{t}^{\infty *}(\omega)}{\partial \theta_{i}}-\frac{\partial l_{t}^{*}(\omega)}{\partial \theta_{i}}\right\| \leq & \frac{1}{\sqrt{T}} \sum_{t=1}^{T} \sup _{\omega}\left\|f_{i t}^{\infty} \varepsilon_{i t}^{\infty *}-f_{i t} \varepsilon_{i t}^{*}\right\| \\
& +\frac{1}{2} \frac{1}{\sqrt{T}} \sum_{t=1}^{T} \sup _{\omega}\left\|\left(\zeta_{i t}^{\infty} \varepsilon_{i t}^{\infty *}-1\right) z_{i t}^{\infty}-\left(\zeta_{i t} \varepsilon_{i t}^{*}-1\right) z_{i t}\right\| \\
= & R_{1 T}+R_{2 T} .
\end{aligned}
$$

Employing similar analysis to that of Halunga and Orme (2009), it can be shown that $E\left[R_{j T}\right]=o(1), j=$ 1,2 , and the result follows by Markov's Inequality. The result that $\frac{1}{\sqrt{T}} \sum_{t=1}^{T} \sup _{\omega}\left|\frac{\partial l_{t}^{\infty *}(\omega)}{\partial \rho_{i j}}-\frac{\partial l_{t}^{*}(\omega)}{\partial \rho_{i j}}\right|=$ $o_{p}(1)$ follows in a similar fashion.

(iii) $E \sup _{\omega}\left\|m_{t}^{\infty}(\omega)\right\|^{2}<\infty$ provided $E \sup _{\omega}\left\|\left(\zeta_{i t}^{\infty} \zeta_{j t}^{\infty}-\rho_{i j}\right) r_{i j, t}^{\infty}\right\|^{2}<\infty$, for $i, j$, which it is by B4.

(iv) Similar to (ii), $\frac{1}{\sqrt{T}} \sum_{t=1}^{T} E \sup _{\omega} \| m_{t}^{\infty}(\omega)-m_{t}(\omega \|=o(1)$, and the result follows by Markov's inequality.

Proof of Proposition 1: $\Sigma^{*}$ is finite by Proposition 6(i) and (iii). As in Ling and McAleer (2003, Lemma 5.2), (i) follows from a Martingale Central Limit Theorem. Part (ii), follows from Proposition 6] and Remark 4

Proof of Proposition 2a By Proposition 1, $\sqrt{T} \bar{m}_{T}(\hat{\omega})=\sqrt{T} \bar{m}_{T}^{\infty}(\hat{\omega})+o_{p}(1)$, so we work with $\sqrt{T} \bar{m}_{T}^{\infty}(\hat{\omega})$ whose limit distribution can be established more easily. Following Ling and McAleer (2003), as adapted by Halunga and Orme (2009), it is straightforward to show that firstly, $\hat{\omega}-\omega_{0}=o_{p}(1)$ and, secondly, that $E \sup _{\omega}\left\|\frac{\partial^{2} l_{t}^{\infty *}(\omega)}{\partial \omega \partial \omega^{\prime}}\right\|<\infty$. Thus a Uniform of Large Numbers yields $T^{-1} \sum_{t=1}^{T} \frac{\partial^{2} l_{t}^{\infty *}(\tilde{\omega})}{\partial \omega \partial \omega^{\prime}}+$ $J_{0}^{*}=o_{p}(1)$, for all $\tilde{\omega}-\omega_{0}=o_{p}(1)$, and $J_{0}^{*}=-E\left[\frac{\partial^{2} l_{t}^{\infty *}(\omega)}{\partial \omega \omega^{\prime}}\right]_{\omega=\omega_{0}}$ is finite and positive definite by Proposition 3 , below. Furthermore, by Proposition $6 \frac{1}{\sqrt{T}} \sum_{t=1}^{T} g_{t}^{\infty *}(\hat{\omega})=o_{p}(1)$, so that a first order asymptotic expansion of this quantity about $\omega_{0}$ yields $\sqrt{T}\left(\hat{\omega}-\omega_{0}\right)=J_{0}^{*-1} \frac{1}{\sqrt{T}} \sum_{t=1}^{T} g_{t}^{\infty *}\left(\omega_{0}\right)+o_{p}(1)$, which is $O_{p}(1)$. Next, it can be shown that $E \sup _{\omega}\left\|\frac{\partial m_{t}^{\infty}(\omega)}{\partial \omega}\right\|<\infty$, for general choice of $r_{i j, t}^{\infty}$, given Assumptions B, so that $\frac{\partial \bar{m}_{T}^{\infty}\left(\omega_{T}\right)}{\partial \omega^{\prime}} \stackrel{p}{\rightarrow}-B_{0}^{*}=E\left[\frac{\partial m_{t}^{\infty}\left(\omega_{0}\right)}{\partial \omega^{\prime}}\right]$, for any sequence $\omega_{T}=\omega_{0}+o_{p}(1)$; see Shadat and Orme (2015). Thus a first order asymptotic expansion of $\bar{m}_{T}^{\infty}(\hat{\omega})$ about $\omega_{0}$ yields

$$
\begin{aligned}
\sqrt{T} \bar{m}_{T}^{\infty}(\hat{\omega}) & =\sqrt{T} \bar{m}_{T}^{\infty}\left(\omega_{0}\right)-B_{0}^{*} \sqrt{T}\left(\hat{\omega}-\omega_{0}\right)+o_{p}(1) \\
& =A_{0}^{*} \frac{1}{\sqrt{T}} \sum_{t=1}^{T} u_{t}^{\infty *}\left(\omega_{0}\right)+o_{p}(1)
\end{aligned}
$$

and, from Proposition $1 . \sqrt{T} \bar{m}_{T}(\hat{\omega}) \stackrel{d}{\longrightarrow} N\left(0, V^{*}\right)$.

\section{Proof of Proposition 3}

(i) Define $z_{t}^{\infty}=\operatorname{diag}\left(z_{i t}^{\infty \prime}\right)$, the $\left(N \times N\left(K+K^{*}\right)\right)$ block diagonal matrix with $z_{i t}^{\infty \prime},\left(1 \times K+K^{*}\right)$, forming the diagonal blocks, and $f_{t}^{\infty}=\operatorname{diag}\left(f_{i t}^{\infty \prime}\right),\left(N \times N\left(K+K^{*}\right)\right)$, constructed in the same way. 
Exploiting the properties of $E_{N}$ and $L_{N}, J_{0}^{*}$ can be obtained by direct differentiation of the scores 36 and (37) which can themselves can be expressed as

$$
\begin{aligned}
\partial l_{t}^{\infty *}(\omega) / \partial \theta & =\frac{1}{4} z_{t}^{\infty \prime}\left(E_{N}^{\prime} P\left(\zeta_{t}^{\infty} \otimes \zeta_{t}^{\infty}\right)-2 \iota_{N}\right)+f_{t}^{\infty \prime} \Gamma^{-1} \zeta_{t}^{\infty} \\
\partial l_{t}^{\infty *}(\omega) / \partial \rho & =\frac{1}{2} L_{N}^{\prime}\left(\Gamma^{-1} \otimes \Gamma^{-1}\right) \operatorname{vec}\left(\zeta_{t}^{\infty} \zeta_{t}^{\infty \prime}-\Gamma\right) .
\end{aligned}
$$

Now,

$$
\begin{aligned}
\partial\left(\zeta_{t}^{\infty} \otimes \zeta_{t}^{\infty}\right) / \partial \theta^{\prime} & =\partial \operatorname{vec}\left(\zeta_{t}^{\infty} \zeta_{t}^{\infty \prime}\right) / \partial \theta^{\prime}=\left(\zeta_{t}^{\infty} \otimes I_{N}+I_{N} \otimes \zeta_{t}^{\infty}\right) \partial \zeta_{t}^{\infty} / \partial \theta^{\prime} \\
\partial \zeta_{t}^{\infty} / \partial \theta^{\prime} & =-f_{t}^{\infty}-\frac{1}{2}\left(\zeta_{t}^{\infty \prime} \otimes I_{N}\right) E_{N} z_{t}^{\infty}
\end{aligned}
$$

and, since $E\left[\zeta_{t}^{\infty} \mid \mathcal{F}_{t-1}\right]_{\omega=\omega_{0}}=0, E\left[I_{N} \otimes \zeta_{t}^{\infty} \mid \mathcal{F}_{t-1}\right]_{\omega=\omega_{0}}=0, E\left[E_{N}^{\prime} P\left(\zeta_{t}^{\infty} \otimes \zeta_{t}^{\infty}\right)-2 \iota_{N} \mid \mathcal{F}_{t-1}\right]_{\omega=\omega_{0}}=0$, and $E_{N}^{\prime} E_{N}+E_{N}^{\prime}\left(\Gamma^{-1} \otimes \Gamma\right) E_{N}=\Gamma_{A}$, we obtain

$$
E\left[\partial^{2} l_{t}^{\infty *}\left(\omega_{0}\right) / \partial \theta \partial \theta^{\prime} \mid \mathcal{F}_{t-1}\right]=-\frac{1}{4}\left[z_{t}^{\infty \prime} \Gamma_{A} z_{t}^{\infty}\right]_{\omega=\omega_{0}}^{\prime}-\left[f_{t}^{\infty \prime} \Gamma^{-1} f_{t}^{\infty}\right]_{\omega=\omega_{0}} .
$$

Similarly,

$$
\begin{aligned}
& E\left[\partial^{2} l_{t}^{\infty *}\left(\omega_{0}\right) / \partial \rho \partial \theta^{\prime} \mid \mathcal{F}_{t-1}\right]=-\frac{1}{4}\left[L_{N}^{\prime} P E_{N} z_{t}^{\infty}\right]_{\omega=\omega_{0}}, \\
& E\left[\partial^{2} l_{t}^{\infty *}\left(\omega_{0}\right) / \partial \rho \partial \rho^{\prime} \mid \mathcal{F}_{t-1}\right]=-\frac{1}{2} L_{N}^{\prime}\left(\Gamma^{-1} \otimes \Gamma^{-1}\right) L_{N}
\end{aligned}
$$

since $\partial \operatorname{vec}(\Gamma) / \partial \rho^{\prime}=L_{N}$. These then yield,

$$
J_{0}^{*}=\frac{1}{4} E\left\{\left[\begin{array}{cc}
z_{t}^{\infty \prime} \Gamma_{A} z_{t}^{\infty} & z_{t}^{\infty \prime} E_{N}^{\prime} P L_{N} \\
L_{N}^{\prime} P E_{N} z_{t}^{\infty} & 2 L_{N}^{\prime}\left(\Gamma^{-1} \otimes \Gamma^{-1}\right) L_{N}
\end{array}\right]\right\}_{\omega=\omega_{0}}+E\left[\begin{array}{cc}
f_{t}^{\infty \prime} \Gamma^{-1} f_{t}^{\infty} & 0 \\
0 & 0
\end{array}\right]_{\omega=\omega_{0}}
$$

which is positive definite; see Shadat and Orme (2015) 14 This expression for $J_{0}^{*}$ concurs with Nakatani and Teräsvirta (2009, p.151), but allowing for regression parameters, $\varphi_{i}$.

From $J_{0}^{*}$ we obtain $J_{T}^{*}(\omega)$ by: $h_{i t}$ replacing $h_{i t}^{\infty}, \omega$ replacing $\omega_{0}$ and $\frac{1}{T} \sum_{t=1}^{T}$ replacing "expectation", throughout; and, noting that $L_{N}^{\prime} P E_{N} \sum_{t=1}^{T} z_{t}=L_{N}^{\prime} P E_{N}\left(I_{N} \otimes \iota_{T}^{\prime}\right) Z=\left(L_{N}^{\prime} P E_{N} \otimes \iota_{T}^{\prime}\right) Z$, $\sum_{t=1}^{T} z_{t}^{\prime} \Gamma_{A} z_{t}=Z^{\prime}\left(\Gamma_{A} \otimes I_{T}\right) Z$ and $\sum_{t=1}^{T} f_{t}^{\prime} \Gamma^{-1} f_{t}=F^{\prime}\left(\Gamma^{-1} \otimes I_{T}\right) F$. Thus $J_{T}^{*}(\hat{\omega})$ is positive definite provided $\hat{Z}$ has full rank of $N\left(K+K^{*}\right)$. Consistency of $J_{T}^{*}(\hat{\omega})$ follows from, e.g., Ling and McAleer (2003) and is verified in Orme and Shadat (2015).

(ii) $B_{0}^{*}=-E\left[\partial m_{t}^{\infty}\left(\omega_{0}\right) / \partial \omega^{\prime}\right]$, is obtained as follows. First, $f_{i t}^{\infty}, z_{i t}^{\infty}, r_{i j, t}^{\infty}$ and $\frac{\partial r_{i j, t}^{\infty}}{\partial \theta_{k}^{\prime}}$ and $\frac{\partial r_{i j, t}^{\infty}}{\partial \rho}$ are all $\mathcal{F}_{t-1}$ measurable. Second, $E\left[\zeta_{0 j t}^{\infty 2} \mid \mathcal{F}_{t-1}\right]=1, E\left[\zeta_{0 j t}^{\infty} f_{0 i t}^{\infty} \mid \mathcal{F}_{t-1}\right]=E\left[\zeta_{0 i t}^{\infty} f_{0 i t}^{\infty} \mid \mathcal{F}_{t-1}\right]=0$, and $E\left[\zeta_{0 i t}^{\infty} \zeta_{0 j t}^{\infty} \mid \mathcal{F}_{t-1}\right]=\rho_{0 i j}$. Then, from previous derivations we obtain

$$
\begin{gathered}
E\left[\partial m_{i j, t}^{\infty}\left(\omega_{0}\right) / \partial \theta^{\prime}\right]=-\frac{1}{2} E\left[\rho_{i j} r_{i j, t}^{\infty}\left(e_{j} \otimes z_{j t}^{\infty}+e_{i} \otimes z_{i t}^{\infty}\right)^{\prime}\right]_{\omega=\omega_{0}}, \\
E\left[\partial m_{i j, t}^{\infty}\left(\omega_{0}\right) / \partial \rho^{\prime}\right]=-E\left[r_{i j, t}^{\infty} e_{i j}^{\prime}\right]_{\omega=\omega_{0}} .
\end{gathered}
$$

\footnotetext{
${ }^{14}$ Ling and McAleer (2003) require that $\Gamma_{A}-I_{N}$ is positive semi-definite. But this seems to arise from an error in their expression for the expected hessian (Ling and McAleer 2003, p.289). Specifically, the error arsises from writing $\left(\partial \operatorname{vec}(\Gamma) / \partial \rho^{\prime}\right)^{\prime}\left(\Gamma^{-1} \otimes \Gamma^{-1}\right) \partial \operatorname{vec}(\Gamma) / \partial \rho^{\prime}$ as $P^{\prime} P$ where, here, $P=\left(I_{N} \otimes \Gamma^{-1}\right) \partial \operatorname{vec}(\Gamma) / \partial \rho^{\prime}$.
} 
The corresponding partitions of $B_{T}^{*}(\omega)$ are thus

$$
\begin{aligned}
B_{i j T}^{*}(\omega) & =\frac{1}{T} \sum_{t=1}^{T}\left[\frac{1}{2} \rho_{i j} r_{i j, t}\left(e_{j}^{\prime} \otimes z_{j t}^{\prime}+e_{i}^{\prime} \otimes z_{i t}^{\prime}\right), r_{i j, t} e_{i j}^{\prime}\right] \\
& =\left[\frac{1}{2} \rho_{i j} R_{i j}^{\prime}\left(e_{j}^{\prime} \otimes Z_{j}+e_{i}^{\prime} \otimes Z_{i}\right), R_{i j}^{\prime}\left(e_{i j}^{\prime} \otimes \iota_{T}\right)\right] .
\end{aligned}
$$

To establish consistency of $B_{T}^{*}(\hat{\omega})$, define $q_{i j, t}^{\infty}=\left(1, z_{i t}^{\infty \prime}, r_{i j, t}^{\infty \prime}\right)^{\prime}$, for any pair $i>j$, and correspondingly $q_{i j, t}=\left(1, z_{i t}^{\prime}, r_{i j, t}^{\prime}\right)^{\prime}$. Then it is immediate from B4 and Remark 3(ii) that: (i) $E \sup _{\omega}\left\|q_{i j, t}^{\infty}\right\|^{2}<\infty$; and, (ii) $\frac{1}{\sqrt{T}} \sum_{t=1}^{T} \sup _{\omega}\left\|q_{i j, t}^{\infty}-q_{i j, t}\right\|=o_{p}(1)$. The result then follows by Lemma 1

Proposition 7 Define $\bar{m}_{T}(\omega)=T^{-1} \sum_{t=1}^{T} m_{t}(\omega)$ constructed from the $\left(q_{i j} \times 1\right)$ sub-vectors $\bar{m}_{i j, T}(\omega)=$ $\frac{1}{T} \sum_{t=1}^{T}\left(\zeta_{i t} \zeta_{j t}-\rho_{i j}\right)\left(r_{i j, t}-\bar{r}_{i j T}(\omega)\right)$ and $\bar{n}_{T}^{\infty}(\omega)=T^{-1} \sum_{t=1}^{T} n_{t}^{\infty}(\omega)$ constructed from the $\left(q_{i j} \times 1\right)$ sub-vectors $\bar{n}_{i j, T}^{\infty}(\omega)=\frac{1}{T} \sum_{t=1}^{T}\left(\zeta_{i t}^{\infty} \zeta_{j t}^{\infty}-\rho_{0, i j}\right)\left(r_{i j, t}^{\infty}-\mu_{i j}\left(\omega_{0}\right)\right)$, where $\mu_{i j}\left(\omega_{0}\right)=E\left[r_{i j, t}^{\infty}\right]_{\omega=\omega_{0}}$ and $\left\|\mu_{i j}(\omega)\right\|<\infty$, by B4. Under Assumptions $A$ and B1, B2:

(i) $E \sup _{\theta}\left\|g_{t}^{\infty}(\theta)\right\|^{2}<\infty$;

(ii) $\frac{1}{\sqrt{T}} \sum_{t=1}^{T} \sup _{\theta}\left\|g_{t}^{\infty}(\theta)-g_{t}(\theta)\right\|=o_{p}(1)$.

In addition, and adding B3 and B4:

(iii) $E \sup _{\omega}\left\|n_{t}^{\infty}(\omega)\right\|^{2}<\infty$

(iv) $\frac{1}{\sqrt{T}} \sum_{t=1}^{T}\left\|n_{t}^{\infty}(\tilde{\omega})-m_{t}(\tilde{\omega})\right\|=o_{p}(1)$, where $\tilde{\omega}$ is the PQML estimator described in Section 2.1.

Proof. It is readily shown that (i) and (ii) hold, and (iii) follows from Proposition 6(iii). For (iv), let $\Delta m_{i j, t}^{\infty}(\omega)=\left(\zeta_{i t}^{\infty} \zeta_{j t}^{\infty}-\rho_{0, i j}\right) r_{i j, t}^{\infty}-\left(\zeta_{i t} \zeta_{j t}-\rho_{0, i j}\right) r_{i j, t}$ and write $\sqrt{T}\left(\bar{n}_{i j, T}^{\infty}(\tilde{\omega})-\bar{m}_{i j, T}(\tilde{\omega})\right)=$ $\frac{1}{\sqrt{T}} \sum_{t=1}^{T} a_{i j, t}(\tilde{\omega})$, where

$$
a_{i j, t}(\omega)=\Delta m_{i j, t}^{\infty}(\omega)+\left(\zeta_{i t} \zeta_{j t}-\rho_{0, i j}\right)\left(\bar{r}_{i j T}(\omega)-\mu_{i j}\left(\omega_{0}\right)\right)-\left(\zeta_{i t}^{\infty} \zeta_{j t}^{\infty}-\zeta_{i t} \zeta_{j t}\right) \mu_{i j}\left(\omega_{0}\right)
$$

Similar to previous arguments, it can be shown that $\frac{1}{\sqrt{T}} \sum_{t=1}^{T}\left\|a_{i j, t}(\tilde{\omega})\right\|=o_{p}(1)$, so that $\sqrt{T}\left\|\bar{n}_{T}^{\infty}(\tilde{\omega})-\bar{m}_{T}(\tilde{\omega})\right\|=$ $o_{p}(1)$.

Proof of Proposition 4 $\Sigma$ is finite by Proposition Proposition 7(i) and (iii). As in Ling and McAleer (2003, Lemma 5.2), (i) follows from a Martingale Central Limit Theorem. Part (ii), follows from Proposition 7 and Remark 4

Proof of Proposition 5: By Proposition 7. $\sqrt{T} \bar{m}_{T}(\tilde{\omega})=\sqrt{T} \bar{n}_{T}^{\infty}(\tilde{\omega})+o_{p}(1)$, and we work with $\sqrt{T} \bar{n}_{T}^{\infty}(\tilde{\omega})$. From the consistency and asymptotic normality of $\tilde{\theta}, \sqrt{T}\left(\tilde{\theta}-\theta_{0}\right)=J_{0}^{-1} \sqrt{T} \bar{g}_{T}^{\infty}\left(\theta_{0}\right)+o_{p}(1)$, where $\sqrt{T} \bar{g}_{T}^{\infty}(\theta)=\sqrt{T} \bar{g}_{T}(\theta)+o_{p}(1)$, by Proposition 7. Similar to proof of Proposition 2, it is readily shown that $E \sup _{\omega}\left\|\frac{\partial n_{t}^{\infty}(\omega)}{\partial \theta}\right\|<\infty$. Thus, a Uniform Law of Large numbers and first order asymptotic expansion of $\sqrt{T} \bar{n}_{T}^{\infty}(\tilde{\omega})$ about $\omega_{0}$, yields $\sqrt{T} \bar{n}_{T}^{\infty}(\tilde{\omega})=A_{0} \sqrt{T} \bar{u}_{T}^{\infty}\left(\omega_{0}\right)+o_{p}(1)$, and the result follows from Proposition 4

\section{References}

Andreou, E., Ghysels, E. (2003). Tests for breaks in the conditional co-movements of asset returns. Statistica Sinica 13(4):1045-1074. 
Aslanidis, N., Osborn, D., Sensier, M. (2008). Co-movements between US and UK stock prices: the roles of macroeconomic information and time-varying conditional correlations. Manchester Economics Discussion Paper Series 0805.

Bera, A. K., Kim, S. (2002). Testing constancy of correlation and other specifications of the BGARCH model with an application to international equity returns. Journal of Empirical Finance 9(2):171-195.

Bera, A. K., McKenzie, C. R. (1986). Alternative forms and properties of the score test. Journal of Applied Statististics 13(1):13-25.

Berkes, I., Horváth, L., Kokoszka, P. (2003). GARCH processes: structure and estimation. Bernoulli 9(2): 201-227.

Billo, M., Caporin, M., Gobbo, M. (2006). Flexible dynamic conditional correlation multivariate GARCH models for asset allocation. Applied Financial Economics Letters 2(2):123-130.

Bollerslev, T. (1990). Modeling the coherence in short-run nominal exchange rates: a multivariate generalized ARCH model. Review of Economics and Statistics 72(3):498-505.

Chesher, A., Spady, R. (1991). Asymptotic expansions of the information matrix test statistics. Econometrica 59(3):787-816.

Davidson, R., MacKinnon, J. G. (1983). Small sample properties of alternative forms of the Lagrange multiplier test. Economics Letters 12(3-4):269-275.

Engle, R. F. (2002). Dynamic conditional correlation: a simple class of multivariate GARCH models. Journal of Business and Economic Statistics 20(3):339-350.

Engle, R. F., Kroner, F. K. (1995). Multivariate simultaneous generalized ARCH. Econometric Theory 11(1):122-150.

Engle, R. F., Ng, V. K. (1993). Measuring and testing the impact of news on volatility. Journal of Finance 48(5):1749-1778.

Engle, R. F., Sheppard, K. (2008). Evaluating the specification of covariance models for large portfolios. Working Paper, Department of Economics, University of Oxford.

Francq, C., Zakoian, J-M. (2004). Maximum likelihood estimation of pure GARCH and ARMA-GARCH processes. Bernoulli 10(4): 605-637.

Hafner, C. M., van Dijk, D., Franses, P.H. (2005). Semi-parametric modelling of correlation dynamics. In: Advances in Econometrics, vol. 20/A. Amsterdam: Elsevier Sciences, pp. 59-103.

Hafner, C. M., Herwartz, H. (2008). Analytical quasi maximum likelihood inference in multivariate volatility models. Metrika 67(2):219-239.

Halunga. A. and Orme, C. D. (2009). First-order asymptotic theory for misspecification tests of GARCH models. Econometric Theory 25(2):364-410.

Kroner, K. F., Claessens, S. (1991). Optimal Dynamic Hedging Portfolios and the Currency Composition of External Debt. Journal of International Money and Finance 10(1):131-148.

Kroner, K. F., Sultan, J. (1991). Exchange rate volatility and time varying hedge ratios. In: PacificBasin Capital Market Research, 2. Amsterdam: North-Holland, pp.397-412. 
Kroner, K. F., Sultan, J. (1993). Time varying distribution and dynamic hedging with foreign currency futures. Journal of Financial and Quantitative Analysis 28(4):535-551.

Lee, J. (2006). The comovement between output and prices: Evidence from a dynamic conditional correlation GARCH model. Economics Letters 91(1):110-116.

Lien, D., Tse, Y. K. (1998). Hedging Time-Varying Downside Risk. Journal of Futures Markets 18(2):705-722.

Lien, D., Tse, Y. K., Tsui, A. K. C. (2002). Evaluating the hedging performance of the constantcorrelation GARCH model. Applied Financial Economics. 12(11):791-798.

Ling, S., McAleer, M. (2003). Asymptotic theory for a vector ARMA-GARCH model. Econometric Theory 19(2):280-310.

Longin, F., Solnik, B. (1995). Is the correlation in international equity returns constant: 1960-1990? Journal of International Money and Finance 14(1):3-26.

Lundbergh, S., Teräsvirta, T. (2002). Evaluating GARCH models. Journal of Econometrics 110(2):417435 .

Magnus, J., Neudecker,H. (1986). Symmetry, 0-1 matrices and Jacobians: a review. Econometric Theory 2:157-190.

Nakatani, T. and Teräsvirta, T. (2009). Testing for volatility interactions in the Constant Conditional Correlation GARCH model. Econometrics Journal 12(1):147-163.

Nelson, D.B., Cao, C.Q. (1992). Inequality constraints in the univariate GARCH model. Journal of Business and Economic Statistics, 10(2):229-235.

Newey, W. K. (1985). Maximum likelihood specification testing and conditional moment tests. Econometrica 53(5):1047-1070.

Orme, C. (1990). The small-sample performance of the information-matrix test. Journal of Econometrics 46(3):309-331,

Park, T., Switzer, L. (1995). Time-varying distribution and the optimal hedge ratios for stock index futures. Applied Financial Economics 5(3):131-137.

Silvennoinen, A., Teräsvirta, T. (2009). Multivariate GARCH models. In: Handbook of Financial Time Series, Springer, pp. 201-229.

Tse, Y. K. (2000). A test for constant correlations in a multivariate GARCH model. Journal of Econometrics 98 (1):107-127.

Tse, Y. K., Tsui, A. K. C. (2002). A multivariate GARCH model with time-varying correlations. Journal of Business and Economic Statistics, 20(3):351-362.

Shadat, W., Orme, C. (2015). Robust Parametric Tests of Constant Conditional Correlation in a MGARCH model: Full Monte Carlo and Detailed Proofs. University of Manchester, EScholarID:264022 (available at https://www.escholar.manchester.ac.uk/uk-ac-man-scw:264022).

Wooldridge, J. (1990). A unified approach to robust, regression-based specification tests. Econometric Theory 6(1):17-43. 
Table 2: CCC Models: Empirical Significance Levels against 5\% nominal level

\begin{tabular}{|c|c|c|c|c|c|c|}
\hline & \multicolumn{6}{|c|}{ AR(1)-CCC-GARCH(1,1) DGP } \\
\hline & \multicolumn{2}{|c|}{ E1 } & \multicolumn{2}{|c|}{ E2 } & \multicolumn{2}{|c|}{ E3 } \\
\hline & $\mathrm{T}=500$ & $\mathrm{~T}=1000$ & $\mathrm{~T}=500$ & $\mathrm{~T}=1000$ & $\mathrm{~T}=500$ & $\mathrm{~T}=1000$ \\
\hline \multicolumn{7}{|c|}{ Gaussian errors } \\
\hline$\widehat{L M}_{T}^{*(o)}$ & 12.98 & 8.52 & 16.07 & 10.18 & 15.65 & 10.31 \\
\hline$\widehat{L M}_{T}^{*(r)}$ & 4.08 & 4.79 & 5.02 & 5.07 & 4.78 & 5.32 \\
\hline$\widetilde{L M}_{T}^{(o)}$ & 7.43 & 6.54 & 3.17 & 4.16 & 3.71 & 4.62 \\
\hline$\widetilde{L M}_{T}^{(r)}$ & 4.03 & 4.61 & 4.56 & 4.76 & 3.99 & 4.52 \\
\hline$\hat{S}_{T}^{* C(o)}$ & 15.33 & 10.01 & 16.75 & 11.61 & 17.81 & 11.77 \\
\hline$\hat{S}_{T}^{* C(r)}$ & 4.32 & 4.70 & 5.11 & 5.87 & 4.82 & 5.65 \\
\hline$\tilde{S}_{T}^{C(o)}$ & 11.84 & 8.71 & 6.57 & 6.82 & 6.81 & 7.08 \\
\hline$\tilde{S}_{T}^{C(r)}$ & 4.37 & 4.61 & 5.02 & 5.61 & 5.09 & 5.76 \\
\hline$\hat{S}_{T}^{* J(o)}$ & 24.74 & 15.04 & 24.93 & 16.10 & 26.15 & 16.77 \\
\hline$\hat{S}_{T}^{* J(r)}$ & 6.40 & 6.10 & 7.15 & 7.06 & 6.26 & 7.03 \\
\hline$\tilde{S}_{T}^{J(o)}$ & 16.92 & 12.04 & 9.78 & 9.40 & 10.54 & 9.99 \\
\hline$\tilde{S}_{T}^{J(r)}$ & 5.77 & 5.95 & 4.82 & 5.09 & 4.37 & 5.16 \\
\hline \multicolumn{7}{|c|}{$t(6)$ errors } \\
\hline$\widehat{L M}_{T}^{*(o)}$ & 20.90 & 11.80 & 27.25 & 19.40 & 27.95 & 20.10 \\
\hline$\widehat{L M}_{T}^{*(r)}$ & 3.10 & 4.00 & 4.80 & 6.90 & 5.75 & 5.75 \\
\hline$\widetilde{L M}_{T}^{(o)}$ & 10.25 & 7.70 & 2.95 & 4.75 & 3.45 & 3.40 \\
\hline$\widetilde{L M}_{T}^{(r)}$ & 3.15 & 4.30 & 4.80 & 6.30 & 4.70 & 4.40 \\
\hline$\hat{S}_{T}^{* C(o)}$ & 26.30 & 16.85 & 32.10 & 24.70 & 30.80 & 23.10 \\
\hline$\hat{S}_{T}^{* C(r)}$ & 4.90 & 4.95 & 5.75 & 7.30 & 5.40 & 6.60 \\
\hline$\tilde{S}_{T}^{C(o)}$ & 15.50 & 10.80 & 8.90 & 9.45 & 7.70 & 8.40 \\
\hline$\tilde{S}_{T}^{C(r)}$ & 4.75 & 4.65 & 4.80 & 6.15 & 4.95 & 5.55 \\
\hline$\hat{S}_{T}^{* J(o)}$ & 46.15 & 32.45 & 46.50 & 34.65 & 46.55 & 33.70 \\
\hline$\hat{S}_{T}^{* J(r)}$ & 7.15 & 7.95 & 7.15 & 9.80 & 7.60 & 8.80 \\
\hline$\stackrel{\stackrel{L}{S}}{T}_{T}^{J(o)}$ & 31.85 & 22.60 & 16.10 & 17.10 & 15.25 & 14.95 \\
\hline$\tilde{S}_{T}^{J(r)}$ & 5.80 & 7.45 & 3.90 & 7.00 & 3.95 & 4.55 \\
\hline
\end{tabular}

Notes:

1. The first block reports results for Tse's LM test, the second and third blocks those for CCM and FCM tests, respectively. Within each block the order is: OPG-FQMLE, ROBUST-FQMLE, OPG-PQMLE, ROBUST-PQMLE.

2. $\mathrm{T}$ is the sample size and results are based on 10,000 simulations for Gaussian errors and 2,000 for $\mathrm{t}(6)$ errors. 


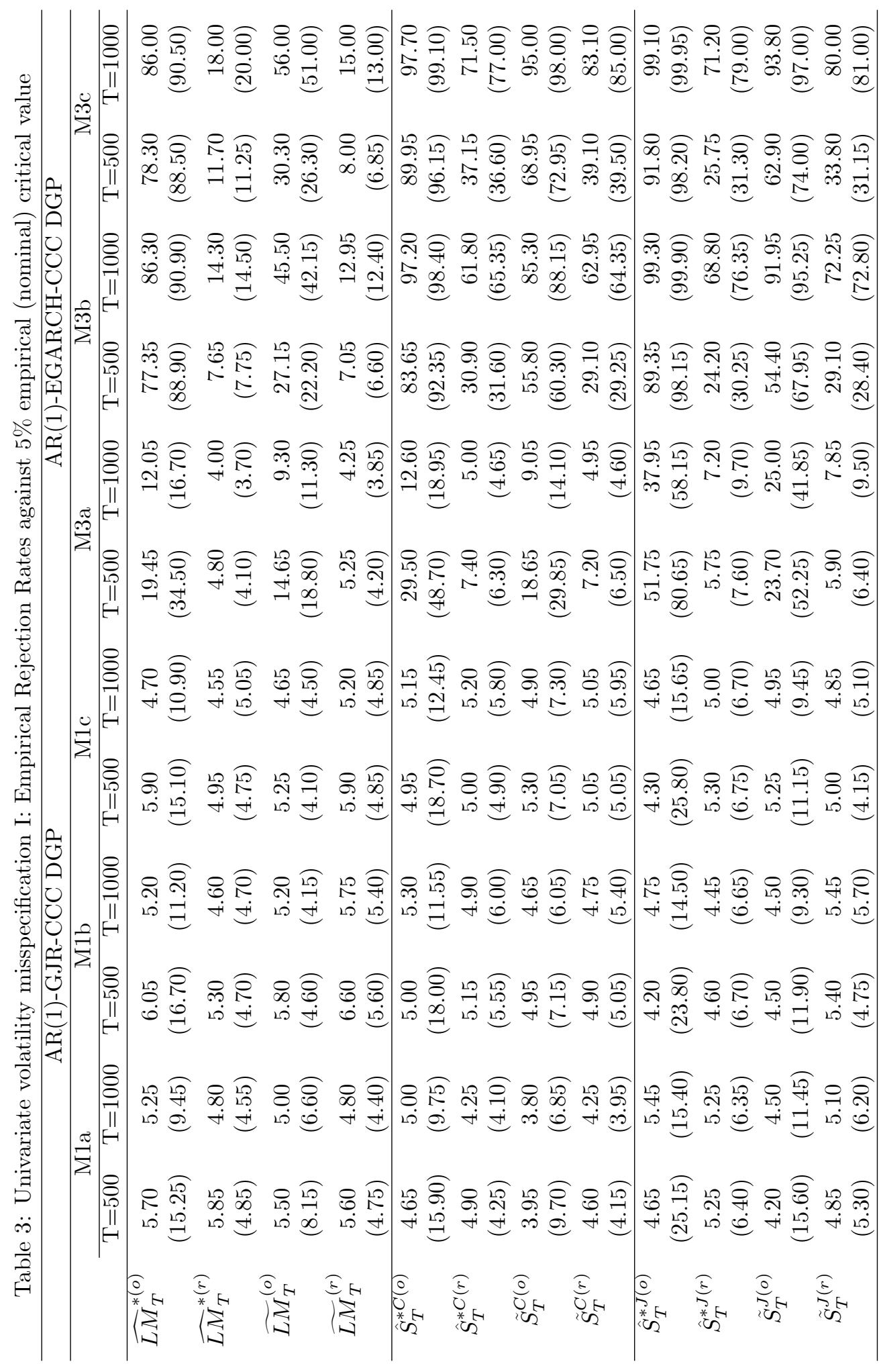


Table 4: DCC Models I: Empirical Rejection Rates against 5\% empirical (nominal) critical value

\begin{tabular}{|c|c|c|c|c|c|c|}
\hline & \multicolumn{6}{|c|}{ AR(1)-DCC-GARCH $(1,1)$ DGP } \\
\hline & \multicolumn{2}{|c|}{$\overline{\mathrm{P} 1}$} & \multicolumn{2}{|c|}{ P2 } & \multicolumn{2}{|c|}{ P3 } \\
\hline & $\mathrm{T}=500$ & $\mathrm{~T}=1000$ & $\mathrm{~T}=500$ & $\mathrm{~T}=1000$ & $\mathrm{~T}=500$ & $\mathrm{~T}=1000$ \\
\hline$\widehat{L M}_{T}^{*(o)}$ & $\begin{array}{c}14.45 \\
(34.85)\end{array}$ & $\begin{array}{c}29.95 \\
(42.75)\end{array}$ & $\begin{array}{c}60.60 \\
(79.30)\end{array}$ & $\begin{array}{c}91.45 \\
(95.00)\end{array}$ & $\begin{array}{c}93.10 \\
(97.35)\end{array}$ & $\begin{array}{c}99.95 \\
(99.95)\end{array}$ \\
\hline$\widehat{L M}_{T}^{*(r)}$ & $\begin{array}{c}14.95 \\
(15.00)\end{array}$ & $\begin{array}{c}27.60 \\
(27.90)\end{array}$ & $\begin{array}{c}51.00 \\
(51.00)\end{array}$ & $\begin{array}{c}88.00 \\
(88.10)\end{array}$ & $\begin{array}{c}85.45 \\
(85.50)\end{array}$ & $\begin{array}{c}99.70 \\
(99.70)\end{array}$ \\
\hline$\widetilde{L M}_{T}^{(o)}$ & $\begin{array}{l}13.90 \\
(9.80)\end{array}$ & $\begin{array}{c}24.85 \\
(27.60)\end{array}$ & $\begin{array}{c}44.00 \\
(37.55)\end{array}$ & $\begin{array}{c}85.60 \\
(83.50)\end{array}$ & $\begin{array}{c}74.80 \\
(69.20)\end{array}$ & $\begin{array}{c}98.75 \\
(98.30)\end{array}$ \\
\hline$\widetilde{L M}_{T}^{(r)}$ & $\begin{array}{l}13.65 \\
(12.85)\end{array}$ & $\begin{array}{l}27.70 \\
(26.60)\end{array}$ & $\begin{array}{c}50.40 \\
(49.10)\end{array}$ & $\begin{array}{c}88.15 \\
(87.60)\end{array}$ & $\begin{array}{c}84.60 \\
(83.45)\end{array}$ & $\begin{array}{c}99.60 \\
(99.60)\end{array}$ \\
\hline$\hat{S}_{T}^{* C(o)}$ & $\begin{array}{c}14.80 \\
(34.00)\end{array}$ & $\begin{array}{c}24.00 \\
(38.90)\end{array}$ & $\begin{array}{c}65.60 \\
(82.45)\end{array}$ & $\begin{array}{c}91.50 \\
(96.40)\end{array}$ & $\begin{array}{c}95.20 \\
(98.60)\end{array}$ & $\begin{array}{c}99.90 \\
(100.00)\end{array}$ \\
\hline$\hat{S}_{T}^{* C(r)}$ & $\begin{array}{c}12.85 \\
(13.10)\end{array}$ & $\begin{array}{l}21.55 \\
(24.00)\end{array}$ & $\begin{array}{c}57.15 \\
(57.50)\end{array}$ & $\begin{array}{c}88.65 \\
(90.20)\end{array}$ & $\begin{array}{c}91.55 \\
(91.60)\end{array}$ & $\begin{array}{c}99.70 \\
(99.75)\end{array}$ \\
\hline$\tilde{S}_{T}^{C(o)}$ & $\begin{array}{c}14.70 \\
(18.45)\end{array}$ & $\begin{array}{c}27.50 \\
(33.10)\end{array}$ & $\begin{array}{c}58.80 \\
(63.65)\end{array}$ & $\begin{array}{c}92.05 \\
(94.10)\end{array}$ & $\begin{array}{c}91.10 \\
(92.45)\end{array}$ & $\begin{array}{c}99.70 \\
(99.80)\end{array}$ \\
\hline$\tilde{S}_{T}^{C(r)}$ & $\begin{array}{c}13.40 \\
(13.45)\end{array}$ & $\begin{array}{l}23.75 \\
(25.15)\end{array}$ & $\begin{array}{c}56.65 \\
(56.65)\end{array}$ & $\begin{array}{c}89.80 \\
(90.55)\end{array}$ & $\begin{array}{c}92.25 \\
(92.30)\end{array}$ & $\begin{array}{c}99.80 \\
(99.80)\end{array}$ \\
\hline$\hat{S}_{T}^{* J(o)}$ & $\begin{array}{c}17.45 \\
(50.95)\end{array}$ & $\begin{array}{l}29.15 \\
(52.45)\end{array}$ & $\begin{array}{c}73.45 \\
(92.00)\end{array}$ & $\begin{array}{c}96.25 \\
(98.90)\end{array}$ & $\begin{array}{c}98.15 \\
(99.85)\end{array}$ & $\begin{array}{c}100.00 \\
(100.00)\end{array}$ \\
\hline$\hat{S}_{T}^{* J(r)}$ & $\begin{array}{c}15.65 \\
(19.25)\end{array}$ & $\begin{array}{l}27.10 \\
(34.00)\end{array}$ & $\begin{array}{c}63.80 \\
(69.80)\end{array}$ & $\begin{array}{c}94.75 \\
(96.05)\end{array}$ & $\begin{array}{c}95.40 \\
(96.15)\end{array}$ & $\begin{array}{c}100.00 \\
(100.00)\end{array}$ \\
\hline$\tilde{S}_{T}^{J(}$ & $\begin{array}{l}17.25 \\
(27.50)\end{array}$ & $\begin{array}{c}32.30 \\
(43.90)\end{array}$ & $\begin{array}{c}65.35 \\
(76.45)\end{array}$ & $\begin{array}{c}95.55 \\
(97.45)\end{array}$ & $\begin{array}{c}94.70 \\
(97.30)\end{array}$ & $\begin{array}{c}100.00 \\
(100.00)\end{array}$ \\
\hline$\tilde{S}_{T}^{J(r)}$ & $\begin{array}{c}15.05 \\
(14.70)\end{array}$ & $\begin{array}{l}25.70 \\
(26.10)\end{array}$ & $\begin{array}{c}61.55 \\
(60.95)\end{array}$ & $\begin{array}{c}94.05 \\
(94.05)\end{array}$ & $\begin{array}{c}94.45 \\
(94.15)\end{array}$ & $\begin{array}{c}100.00 \\
(100.00)\end{array}$ \\
\hline
\end{tabular}

Notes:

1. $\mathrm{T}$ is the sample size and results are based on 2,000 simulations.

2.For each test the first (second) row report rejection rates using empirical (nominal) critical values; i.e., figures in the first row for ecah test statistic report size-adjusted rejection rates 